\title{
Atrial remodeling and subsequent atrial tachyarrhythmias
}

\author{
PhD Thesis
}

by

Mária Kohári, M.D.

Szeged

2016 


\title{
Atrial remodeling and subsequent atrial tachyarrhythmias
}

\author{
PhD Thesis
}

by
Mária Kohári, M.D.

Supervisor:

László Sághy, M.D., Ph.D.

$2^{\text {nd }}$ Department of Internal Medicine and Cardiology Centre

Faculty of Medicine, University of Szeged

Doctoral School of Clinical Medicine

Clinical and Experimental Cardiovascular Science

2016 


\section{Content}

Publications related to the subject of the Thesis ................................................................ 1

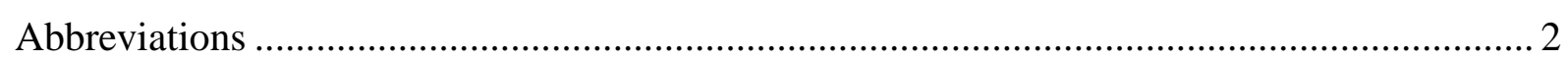

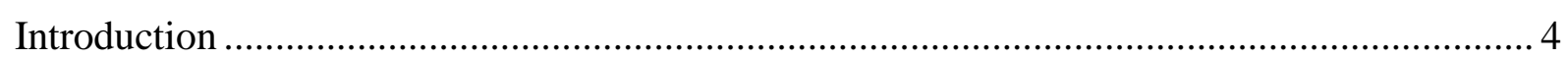

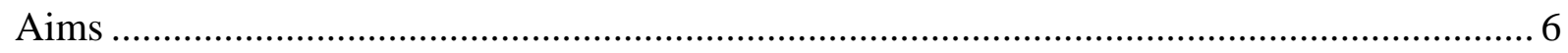

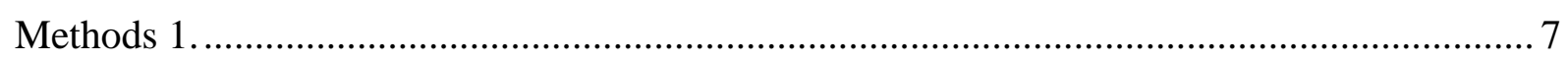

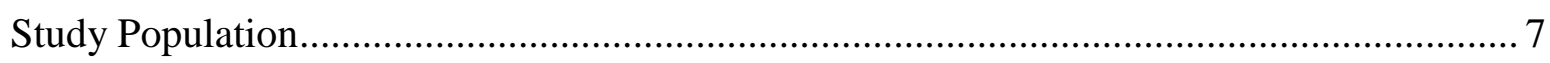

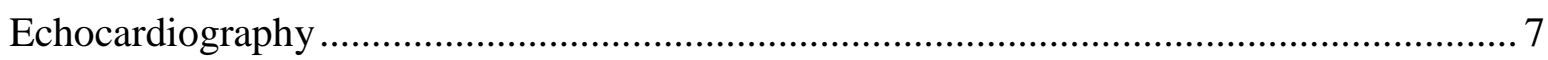

Catheter Ablation and left atrial pressure (LAP) recordings .......................................... 8

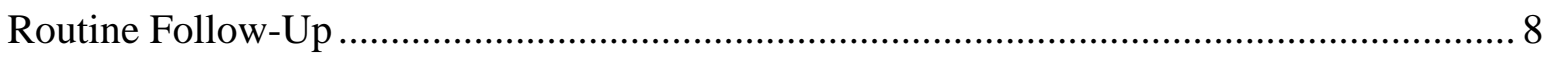

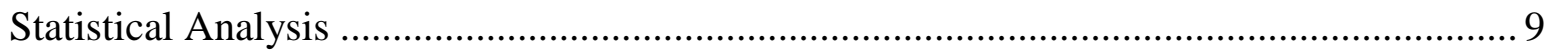

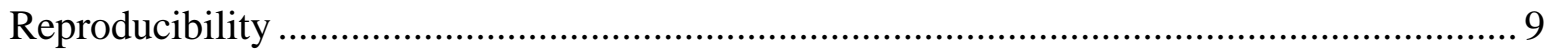

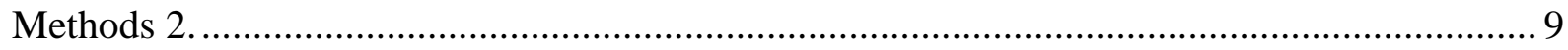

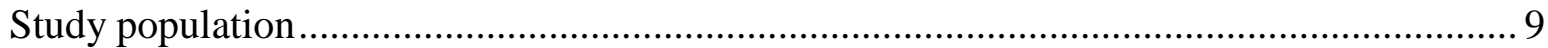

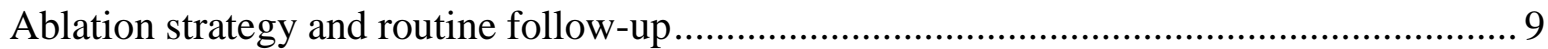

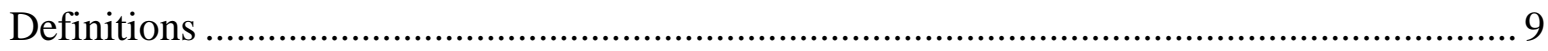

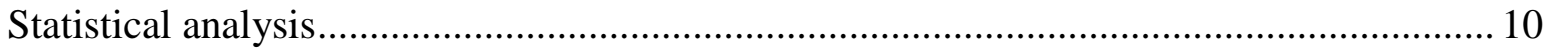

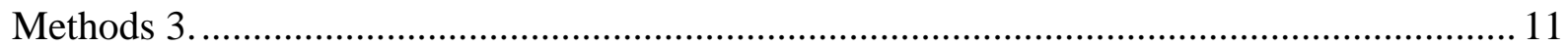

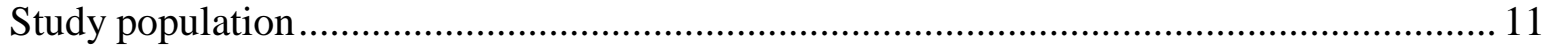

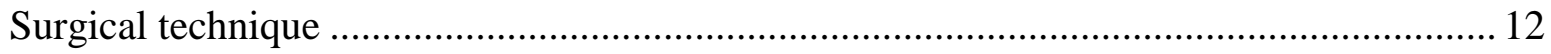

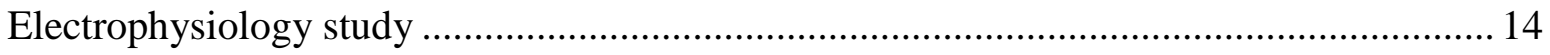

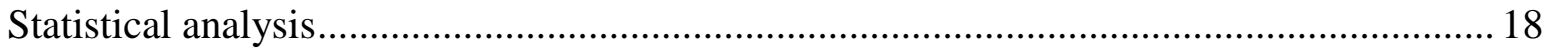

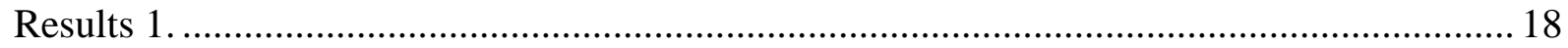

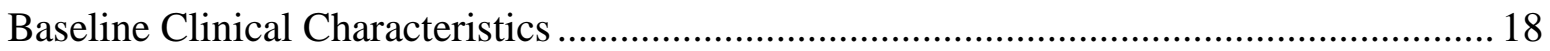

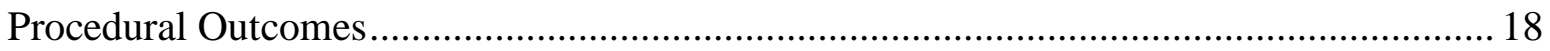

Clinical Parameters Associated with Recurrence ............................................................. 19

Echocardiographic and Atrial Pressure Parameters ........................................................... 21

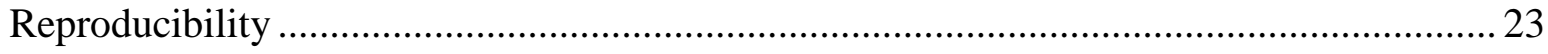

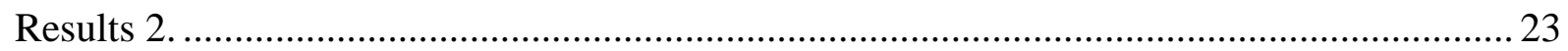

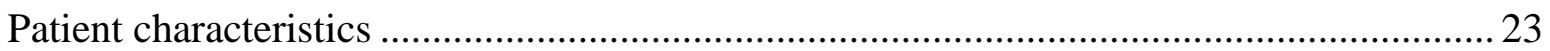

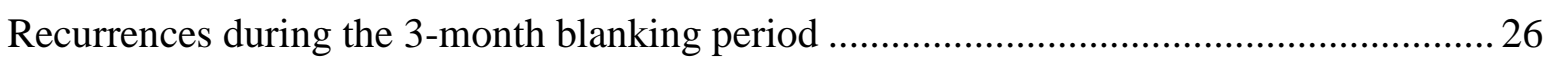

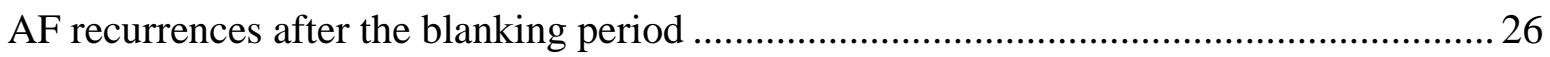

Efficacy of antiarrhythmic drug therapy after recurrence ............................................. 29

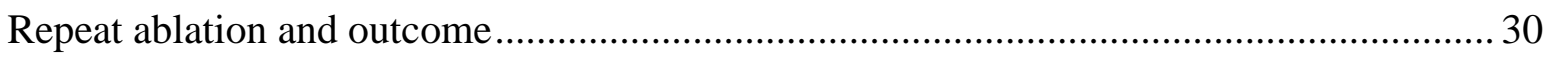

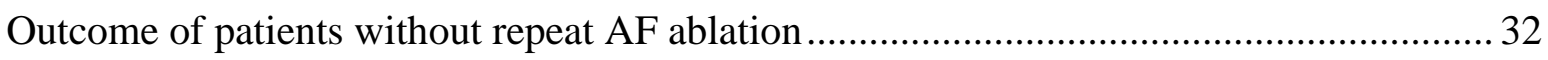


Outcomes in paroxysmal and nonparoxysmal AF.

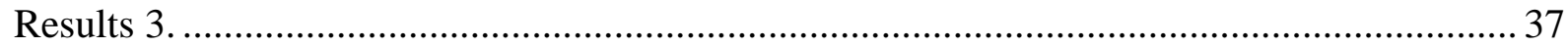

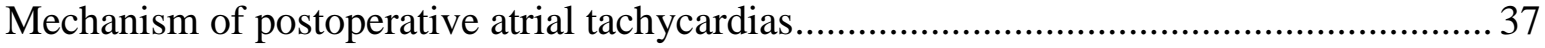

Distribution of atrial tachycardias between surgical groups .............................................. 37

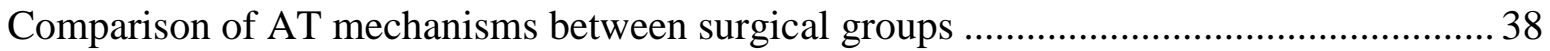

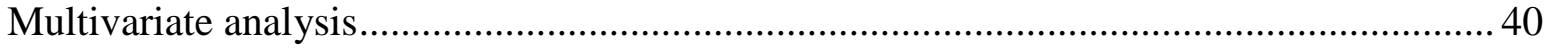

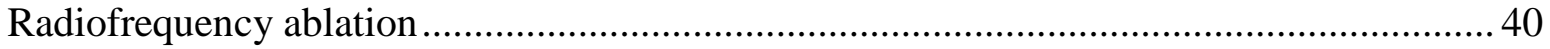

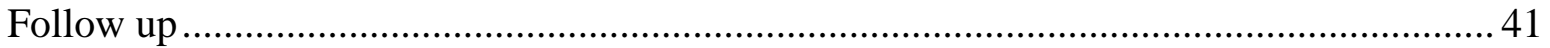

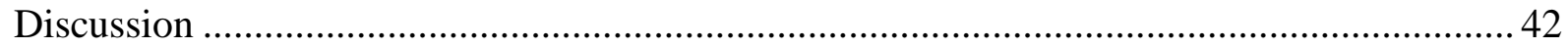

1 1.

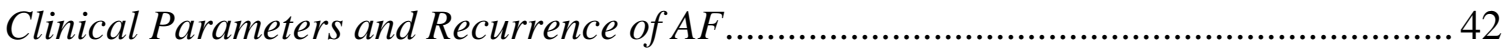

Echocardiographic Parameters and Recurrence of AF ..................................................... 42

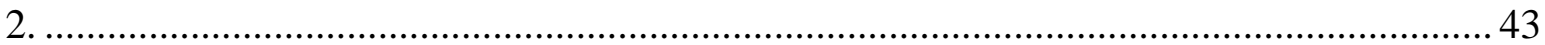

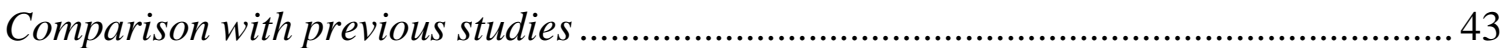

Other factors associated with the time of recurrence after AFablation and clinical

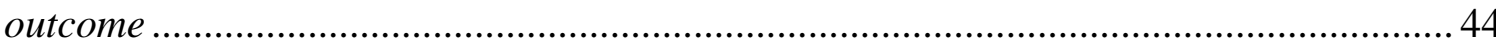

Mechanistic implications for the influence of the time of recurrence after ablation on subsequent clinical outcome ...................................................................................... 45

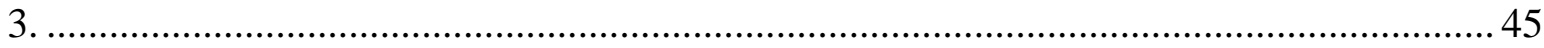

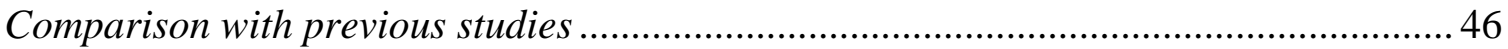

Cavotricuspid isthmus-dependent postoperative flutter .................................................. 46

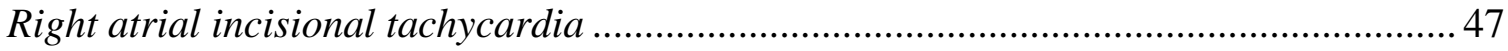

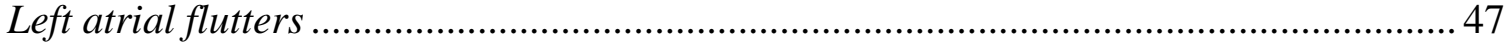

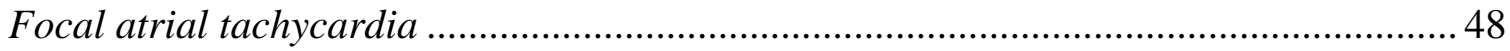

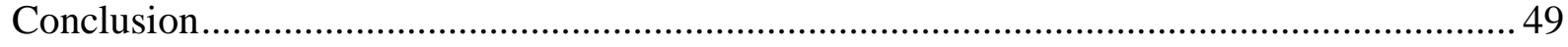

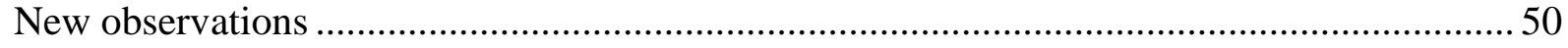

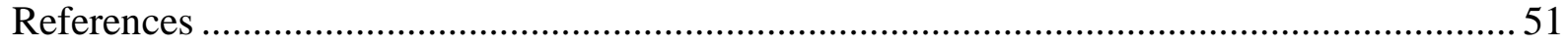

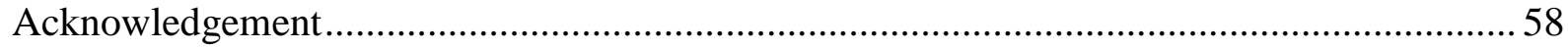

Reprints of published papers 


\section{Publications related to the subject of the Thesis}

I. Kohári M., Zado E. S. , Marchlinski F. E. , Callans D. J. , Han Y., Left Atrial Volume Best Predicts Recurrence after Catheter Ablation in Patients with Persistent and Longstanding Persistent Atrial Fibrillation, PACE 2014; 37:422-429 IF: 1.129

II. Gaztanãga L., Frankel D. S. , Kohari M., Kondapalli L., Zado E. S. ,Marchlinski F. E., Time to recurrence of atrial fibrillation influences outcome following catheter ablation, Heart Rhythm 2013;10:2-9 IF: 4.918

III. Pap R. , Kohári M., Makai A. , Bencsik G. , Traykov B.V., Gallardo R., Klausz G., Kis Zs., Forster T., Sághy L. Surgical technique and the mechanism of atrial tachycardia late after open heart surgery, J Interv Card Electrophysiol, 2012 IF: 1.386 


\section{Abbreviations}

ASD - atrial septal defect

VSD - ventricular septal defect

PAPVC - partial anomalous pulmonary venous connection

CHD - coronary heart disease (significant stenosis or prior myocardial infarction)

LA - left atrium, RA - right atrium

EDD - end-diastolic left ventricular diameter

ESD - end-systolic left ventricular diameter

$\mathrm{EF}$ - left ventricular ejection fraction

PPI - post-pacing interval after entrainment

TCL - tachycardia cycle length

$\mathrm{AF}$ - atrial fibrillation

AFL -atrial flutter

CTId - cavostricuspid isthmus dependent

PVI - pulmonary vein isolation

AAD - antiarrhythmic drug

ACE - angiotensin-converting enzyme

BSA - body surface area

COPD - chronic obstructive pulmonary disease

ICD - implantable cardioverter defibrillator

LVEF - left ventricular ejection fraction.

2C - 2-chamber view

4C - 4-chamber view

E - early diastolic peak transmitral flow velocity

E' lateral, septal, avg - annular velocity at lateral, septal side, and the average of them

TDI - tissue Doppler imaging

PWD - pulsed-wave Doppler

LAA - LA area

LAV - LA volume

LAVi - LA volume indexed to body surface area

LAVmax - LA maximum volume

LAVmin - LA minimum volume

PVD - pulmonary vein diastolic flow velocity 
PVS - pulmonary vein systolic flow velocity

PVSD - ratio of PVS and PVD

$\mathrm{CM}$ - cardiomyopathy

CI - confidence interval

DCCV - direct current cardioversion

OR - Odds ratio 


\section{Introduction}

Treatment of atrial tachycardias has changed a lot in the last decade; catheter ablation has emerged as the curative therapy. In order to achieve the better outcome, clinical arrhythmologists are paying more attention to the anatomy and function of the atrial structure. Being familiar with atrial anatomy, structure and function is particularly important to treat patients with atrial tachycardias. Out of these atrial tachycardias, atrial fibrillation (AF) is the most common arrhythmia in the clinical practice and is associated with several important adverse clinical outcomes, including impairment of quality of life, embolic events and congestive heart failure. ${ }^{1}$ Atrial fibrillation itself produces changes in atrial function and structure which could play a role in the development of these clinical consequences. According to experimental studies, episodes of AF generate even longer AF episodes and continuous atrial remodeling. ${ }^{2}$ This remodeling manifests in several levels such as electrical, structural and contractile remodeling. All of these components can create a vicious circle. After the arrhythmia has been triggered, it affects the ion currents and ion channels at first and creates electrical remodeling. Contractile remodeling refers to a reduction in atrial contraction with an increased wall tension and finally atrial dilatation which is the hallmark of remodeling. The existence of atrial fibrillation not only changes the electrical activity of the atria, but it has a strong influence on the ultrastructure of the chamber.

Pulmonary vein isolation (PVI) using radiofrequency catheter ablation (RFA) is an important and effective therapeutic option for $\mathrm{AF}^{3}$ Since Haissaguere's discovery, the pulmonary vein isolation has become a well-established method for the treatment of either paroxysmal or persistent atrial fibrillation. ${ }^{4}$ Several technical improvements and advances have been introduced in the last two decade, but AF recurrences are common after the procedures, a significant number of patients require repeated attempts. The recurrence rate of AF after catheter ablation is reported to be as high as $15-50 \%$ depending on the ablation strategy and the AF type. ${ }^{3,5}$ Most recurrences occur in the first 6 months after ablation, but even after 1 year, an annual additional recurrence rate of 5-9\% has been described. ${ }^{6,7}$ Awareness of these results and careful patient selection are really important to target the most appropriate population with the previously mentioned approach.

Left atrial (LA) dimension, left ventricular (LV) ejection fraction and valvulopathies can influence AF recurrences. The left atrium has a significant functional role in cardiac 
performance by varying left ventricular filling with its reservoir, conduit and contractile functions. ${ }^{8}$ With the noncompliance of the LV, LA pressure rises to maintain adequate LV filling and the subsequent atrial wall tension leads to chamber dilatation, stretching of the atrial myocardium and longstanding elevation of afterload, which finally causes remodeling of the LA. ${ }^{9}$ Left atrial volume increases with the progression of diastolic dysfunction, consequently the LA volume influences the LV filling pressures over time. ${ }^{10}$ Despite the numerous data in the literature, the association between clinical and diastolic parameters and recurrence after catheter ablation in atrial fibrillation is not fully described. In last few years, many new echocardiographic parameters have been described to characterize the diastolic function in patients with AF. Patients with AF recurrence had significantly larger LA size, increased early diastolic transmitral flow velocity (E) and increased E/E' (ratio of early diastolic peak transmitral inflow to early diastolic peak mitral annular velocity). ${ }^{11,12}$ Indexed LA volume (LAV) was shown to be strongly associated with AF recurrence after catheter ablation in a cohort of patients with paroxysmal and persistent AF. ${ }^{13,14}$ Among the tissue Doppler imaging (TDI) parameters, the $\mathrm{E} / \mathrm{E}^{\prime}$ is the key parameter in the estimation of the degree of diastolic dysfunction and this parameter was evaluated for AF recurrence after electrical cardioversion ${ }^{15}$ and catheter ablation. ${ }^{11}$ Based on these results, there is no single echo parameter which can be able to clearly predict AF occurrence and identify patients who will benefit from catheter ablation.

Atrial flutter often coexists with atrial fibrillation. It can produce atrial electrical remodeling similarly as $\mathrm{AF}^{16}$ and probably is the most common atrial arrhythmia in patients who undergo open heart surgery. Several types of atrial flutters may develop in this very special subgroup of patients and the strategy of treatment is different compared to patients with typical flutter without heart surgery. Heart surgery for acquired valvular problems or ischemic heart disease involves incisions applied to the right and/or left atrium either for establishing extracorporeal circulation or for approaching the intracardiac structures (coronary sinus, interatrial or interventricular septum, atrioventricular valves, etc.). ${ }^{17}$ These atriotomies create an ideal substrate for atrial tachycardias (AT) together with structural and hemodynamic changes resulting from the underlying heart disease. These two factors have been thought to play a major role in the development of atrial tachycardia (AT) months or even years after surgery. ${ }^{18-22}$ Detailed knowledge of the underlying anatomy, surgical techniques and incisions are critical in understanding the mechanism of the subsequent arrhythmia. Besides the most common cavotricuspid-isthmus (CTI)-dependent atrial flutter 
(AFL), atypical AFLs are also not uncommon in these population of patients. ${ }^{23,24}$ AT mechanisms can be different depending on the underlying heart disease, the type of the operation, and specifically the type of atrial incision applied during the surgery. Due to the complexity of multiple AFL circuits, an empirical approach has been suggested in sinus rhythm in patients with previous heart surgery and coexistent atrial incisions. ${ }^{18-20,25}$

\section{Aims}

1. According to the observations associated with AF and diastolic dysfunction, we sought to evaluate atrial diastolic function parameters including two-dimensional (2D), pulsed-wave Doppler (PWD) and TDI parameters, and the directly measured left atrial pressure (LAP) values for associations with AF recurrence after RFA in a large cohort of patients with persistent and longstanding persistent AF.

2. We sought to perform a long-term follow-up study of patients with AF recurrence after catheter ablation to determine whether the time to recurrence of AF influenced AF characteristics, response to therapy and clinical outcome.

3. We sought to establish an association between organic heart disease, atrial incisions, and the mechanism of AT developing late after open heart surgery in a routinely encountered patient population with acquired or simple congenital heart disease. 


\section{Methods 1.}

\section{$\underline{\text { Study Population }}$}

The study protocol was approved by the Institutional Review Board at our institution with waiver of consent for retrospective review of medical records. There were 493 patients with drug refractory persistent and longstanding persistent AF who underwent PVI by RFA at the Hospital of the University of Pennsylvania between April 2009 and April 2011. The patients were excluded from analysis if they had previous catheter ablation (251 patients), a history of open heart surgery (17 patients), moderate or severe mitral regurgitation (14 patients), reduced LV ejection fraction $(\mathrm{EF})(<50 \%, 30$ patients), mitral annular calcification (five patients), pacemaker dependency (four patients), no LA pressure registration (25 patients), were in AF the following day after catheter ablation (seven patients), and less than 6 months follow-up (21 patients). Persistent AF was defined as continuous AF for greater than 7 days or cardioversion after 48 hours of continuous AF. Longstanding persistent AF was defined as continuous $\mathrm{AF}$ for greater than 12 months. ${ }^{26}$ Clinical data were obtained from medical records.

\section{Echocardiography}

All patients underwent routine clinical transthoracic echocardiogram (TTE) examinations including M-mode, 2D, PWD and TDI on the first postprocedural day following PVI. All studies were analyzed in a blinded fashion on dedicated workstations (ProSolv CardioVascular Client version 4.0.4) by a single reader. Left atrial diameter was obtained in the parasternal long axis view. Left atrial area (LAA) and left atrial length were measured in the apical 4-chamber (4C) view and apical 2-chamber (2C) view. LAV was derived using the biplane area-length method. Both LAA and LAV were measured at LV end-systole (LA maximum volume, LAVmax; LA maximum area, LAAmax) and at LV end-diastole (LA minimum volume, LAVmin; LA minimum area, LAAmin). LAV index (LAVi) was calculated based on the body surface area. Mitral inflow measurements using PWD included peak early diastolic velocity (E) and deceleration time (DT) of early diastolic velocity. Late diastolic velocity (A) is not obtained due to generally diminutive velocities 1 day postablation. Pulmonary venous flows using PWD were characterized by peak systolic velocity (PVS), peak diastolic velocity (PVD), and the ratio of PVS to PVD (PVSD). TDI obtained from the $4 \mathrm{C}$ view included early velocities from the septal and lateral mitral annulus 
(E'septal and E'lateral, respectively). The ratio of early diastolic transmitral flow velocity to annular motion velocity was calculated for both lateral and septal annular sites (E/E'lateral and E/E'septal, respectively) and was also calculated for the average between the two sites (E/E'average).

\section{Catheter Ablation and left atrial pressure (LAP) recordings}

PVI was performed per routine at our institution as previously described. ${ }^{27,28}$ Briefly, multipolar catheters were placed in the coronary sinus and posterior right atrium (RA) and a diagnostic intracardiac ultrasound catheter was advanced to the RA. Two transseptal punctures were performed through which the ablation and circular mapping catheters were advanced into the LA. A bolus of unfractionated heparin was administered prior to the first transseptal puncture and infusion was titrated to maintain activated clotting time of $>350$ seconds for the duration of the procedure. Immediately after the transseptal access, the LA pressure was transduced through the transseptal needle. LA X-wave pressure nadir, LA peak v-wave pressure, and mean LA pressure were recorded using an electrophysiologic recording system (Prucka-GE, Houston, TX, USA). The ablation endpoints were absence of PV potential recordings of the circular mapping catheter, which defined entrance block; failure to capture the LA when pacing from all bipoles of the circular mapping catheter (output $10 \mathrm{~mA}$, pulse width $2 \mathrm{~ms}$ ), defined exit block ${ }^{29}$; and no AF with incremental infusion of up to $20 \mu \mathrm{g} / \mathrm{min}$ of isoproterenol. Additional ablation procedures were performed as clinically appropriate by the electrophysiologists.

\section{$\underline{\text { Routine Follow-Up }}$}

All patients underwent a TTE the day after the procedure. Patients were typically restarted on previous antiarrhythmic drugs prior to discharge and continued during follow-up at the discretion of the clinician. Patients had outpatient visits at 6-10 weeks and 6 months, and the majority of patients had visits at 1 year from the date of the ablation procedure. Patients routinely underwent a 4-week period of transtelephonic monitoring immediately after ablation and at least once again at 3-12 months to assess for asymptomatic AF recurrence. At each outpatient visit, patient symptoms were assessed and a 12-lead electrocardiogram (ECG) was recorded. Beyond the 1-year period, the patients were either seen at our institution or by their local cardiologist based on their preferences but followed with telephone calls from our center. 


\section{$\underline{\text { Statistical Analysis }}$}

Statistical analyses were performed using STATA software (version 10, StataCorp, College Station, TX, USA). Continuous variables were expressed as mean \pm standard deviation and categorical variables were expressed as percentages. Comparison of data between the two groups with and without recurrence was performed using a two-tailed, unpaired Student's ttest for continuous variables and $\chi^{2}$ test for the categorical variables. Logistic regression analysis was used to identify univariate and multivariable parameters of AF recurrence. Note that $\mathrm{P}$ values of $\leq 0.05$ were considered statistically significant.

\section{$\underline{\text { Reproducibility }}$}

Fifteen randomly selected TTE studies were reviewed by another independent blinded observer to assess interobserver variability. The same 15 studies were reinterpreted by the original reader in a blinded fashion 3 months after the initial reading to assess intraobserver variability.

\section{Methods 2.}

\section{Study population}

We identified patients who (1) underwent first catheter ablation for AF at the Hospital of the University of Pennsylvania between 2004 and 2008, (2) had recurrent AF after ablation, and (3) had a minimum of 18-month follow-up after recurrence. Patients were divided into an early (E) recurrence group, with recurrences occurring 3-6 months of ablation, a late (L) group, with recurrences occurring between 6 and 12 months, and a very late (VL) group, with recurrences occurring $>1$ year after ablation.

\section{Ablation strategy and routine follow-up}

PVI and routine follow-up was performed as previously described. ${ }^{27-31}$

\section{$\underline{\text { Definitions }}$}

Because AF that occurs very early after ablation may be transient, AF recurrences occurring within the first 3 months of ablation were excluded. In contrast, documented episodes of AF or any organized atrial arrhythmia lasting > 30 seconds after the 3-month blanking period 
were counted as AF recurrences. In order to facilitate analysis, the arrhythmia burden after the initial recurrence was dichotomized as being rare only if during every 6-month follow-up window, no $\mathrm{AF}$ or $\leq 2 \mathrm{AF}$ episodes occurred and $\leq 1$ direct current cardioversion (DCCV) was performed. Patients were considered drug responders if after initiating new drug therapy because of AF recurrence the patient had no symptomatic AF with at least 6 months of additional clinical follow-up and at least one > 2-week TTM showing no recurrence. After a repeat $\mathrm{AF}$ ablation procedure, $\mathrm{AF}$ control was defined as $\mathrm{AF}$ free or rare $\mathrm{AF}$ episodes in each year of follow-up (12-month minimum) with at least 1 extended period of transtelephonic ECG monitoring. For those patients with rare episodes before repeat ablation, a more stringent definition of AF control was used after repeat ablation (no or only 1 episode of AF during the follow-up of at least 1 year).

\section{$\underline{\text { Statistical analysis }}$}

Continuous variables are expressed as mean \pm SD and categorical variables as number (percentages). The analysis of variance test was used to compare continuous variables across multiple groups. The $\chi^{2}$ test with Yates correction or Fisher exact test was used to analyze categorical variables. Multivariate logistic regression was utilized to identify predictors of favorable clinical outcomes after the initial recurrence. Variables included in Table 8 in addition to recurrences during the blanking period, DCCV during the blanking period, on AADs at the time of recurrence, and rare episodes after the blanking period were subjected to univariate screening. Variables showing marginal associations with favorable outcome $(\mathrm{P}<0.10)$ were assessed in multivariate models. A 2-tailed $\mathrm{P}$ value of $\leq 0.05$ was considered statistically significant. All statistical analyses were performed by using SPSS version 16.0 (SPSS Inc, Chicago, IL). 


\section{Methods 3.}

\section{Study population}

Consecutive patients undergoing electrophysiology study for documented AT at least 3 months after open heart surgery for acquired or simple congenital heart disease were enrolled. Patients after complex corrective surgery (Senning, Mustard or Fontan procedure) were not included. Patients undergoing empirical CTI ablation (surface ECG documentation of typical flutter, ablation during sinus rhythm), non-inducible tachycardia or with previous surgical or catheter based ablation were excluded. Altogether one hundred patients were enrolled, clinical characteristics are summarized in Table 1. 


\begin{tabular}{|ll|}
\hline Characteristics (N=100) & \\
\hline Mean age (range), years & $64(23-89)$ \\
Male/female & $61 / 39$ \\
Months since surgery & \\
$\quad$ Median & 60 \\
$\quad$ Min-max & $3-274$ \\
Congenital heart disease & \\
$\quad$ ASD & 13 \\
$\quad$ VSD & 2 \\
$\quad$ Ebstein & 2 \\
$\quad$ Pulm. stenosis & 1 \\
$\quad$ Fallot & 2 \\
$\quad$ PAPVC & 2 \\
Acquired heart disease & \\
$\quad$ CHD & 31 \\
$\quad$ Valvular & 83 \\
$\quad$ Myxoma & 4 \\
Hypertension & 62 \\
Diabetes & 21 \\
LA diameter (mm) & $52 \pm 9$ \\
EDD (mm) & $56 \pm 9$ \\
ESD (mm) & $39 \pm 10$ \\
EF (\%) & $56 \pm 14$ \\
\hline
\end{tabular}

Table 1. Clinical caracteristics of patients

ASD: atrial septal defect, VSD: ventricular septal defect, PAPVC: partial anomalous pulmonary venous connection, CHD: coronary heart disease (significant stenosis or prior myocardial infarction), valvular heart disease moderate or greater regurgitation or significant stenosis, LA: left atrium, EDD: end-diastolic left ventricular diameter, ESD: endsystolic left ventricular diameter, EF: left ventricular ejection fraction

\section{$\underline{\text { Surgical technique }}$}

Surgical procedures were grouped according to the incisions applied to the atria. In some cases, only a right atrial (RA) incision was placed: either for venous cannulation for cardiopulmonary bypass via a small incision on the RA appendage or a long incision on the RA free wall for the placement and fixation of a cardioplegic cannula inside the coronary sinus (open technique for retrograde cardioplegia) or to perform surgery at the right side of the heart. Venous cannulas for cardiopulmonary bypass in the latter cases were placed through incisions on the superior caval vein and the inferior vena cava-RA junction (bicaval 
cannulation). In some patients, both RA and left atrial (LA) incisions were placed: The RA free wall atriotomy was prolonged superiorly and onto the interatrial septum to access the left atrium (transseptal LA atriotomy). The remaining patients had only LA operation using direct LA atriotomy (in the interatrial groove) along with bicaval cannulation (Fig. 1, Table 2).

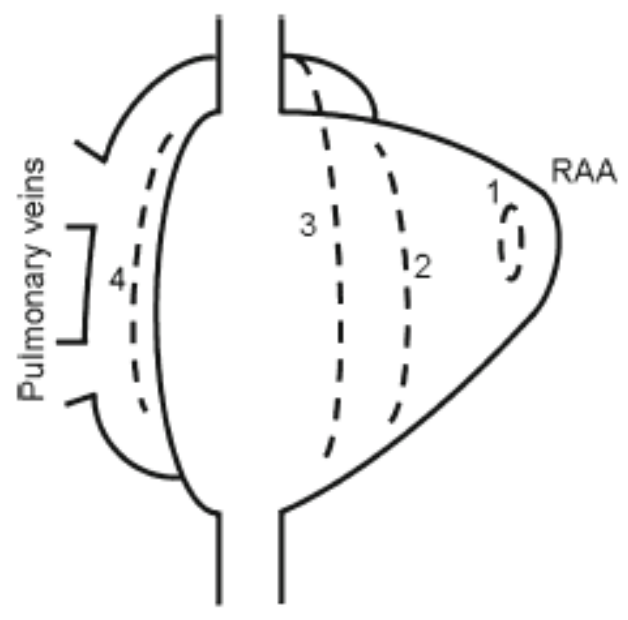

Figure 1. Schematic drawing of the different atrial incisions applied: 1 right atrial appendage cannulation, 2 right atrial free wall atriotomy, 3 transseptal left atriotomy, 4 direct left atriotomy 


\begin{tabular}{|c|c|c|}
\hline Atrial incision & Operation & $\mathbf{N}$ \\
\hline \multirow[t]{4}{*}{ RA appendage cannulation } & CABG & 9 \\
\hline & Ao & 7 \\
\hline & CABG and Ao & 3 \\
\hline & $\mathrm{Pu}$ & 1 \\
\hline \multirow[t]{4}{*}{ RA free wall atriotomy } & Retrograde cardioplegia (CABG and/or Ao) & 14 \\
\hline & ASD & 13 \\
\hline & VSD & 2 \\
\hline & $\operatorname{Tr}$ & 3 \\
\hline \multirow[t]{5}{*}{ Transseptal LA atriotomy } & $\mathrm{Mi}$ & 20 \\
\hline & $\mathrm{Mi}$ and $\mathrm{CABG}$ & 8 \\
\hline & Mi and Ao & 5 \\
\hline & Mi and $\mathrm{Tr}$ & 4 \\
\hline & LA myxoma & 4 \\
\hline \multirow[t]{2}{*}{ Direct LA atriotomy } & $\mathrm{Mi}$ & 6 \\
\hline & Mi and Ao & 1 \\
\hline
\end{tabular}

Table 2. Surgical procedures, CABG coronary artery bypass graft, Ao aortic valve, $\mathrm{Pu}$ pulmonary valve, ASD atrial septal defect, VSD ventricular septal defect, Tr tricuspid valve, Mi mitral valve, RA right atrial, LA left atrial

\section{Electrophysiology study}

Patients were studied under light sedation using midazolam \pm fentanyl. Multipolar catheters were introduced through the femoral \pm the internal jugular or the subclavian vein. One catheter was positioned in the coronary sinus (CS) with the most proximal bipole at the ostium. Another catheter was placed in the RA around the tricuspid annulus, with the most distal electrode in the low RA. A steerable catheter was used for mapping and pacing from other locations. Radiofrequency ablation was carried out by an externally irrigated ablation catheter (NaviStar ThermoCool, Biosense WebsterInc., Diamond Bar, CA, USA or Therapy Cool Path, St. Jude Medical Inc., St. Paul, MN, USA). During sinus rhythm, atrial extrastimulation was carried out at two drive cycle lengths, using at least two extrastimuli. This was repeated after ablation of every tachycardia. Only sustained (> 30 s) tachycardias amenable for detailed mapping are included in this analysis. Non-sustained, irregular (> $30 \mathrm{~ms}$ cycle length variation) tachycardias, and those that terminated or transformed during mapping were excluded. Activation mapping during AT was accomplished by multielectrode recording and in cases of non-CTI-dependent AT, use of an 
electroanatomic mapping system (CARTO ${ }^{\mathrm{TM}} \mathrm{XP}$, Biosense Webster Inc.). Entrainment pacing was performed with a cycle length (CL) 10-30 ms shorter than the tachycardia CL. The pacing site was considered to be in the reentrant circuit if the post-pacing interval, measured from the pacing stimulus to the first non-paced electrogram recorded by the pacing electrode, was less than $30 \mathrm{~ms}$ longer than the tachycardia CL. Atrial tachycardias were classified as macro-reentrant (flutter) if distant atrial sites were determined to be part of the reentry circuit, and continuous atrial activation was demonstrated by activation mapping. Focal ATs were defined by centrifugal activation from a discrete source. Atrial flutter (AFL) was classified as CTI-dependent if entrainment mapping determined the CTI to be part of the circuit and the flutter terminated during ablation at the CTI. If the above were not fulfilled, the AFL was labeled non-CTI-dependent and further classified as RA incisional tachycardia, if the RA free wall was part of the circuit based on entrainment, a RA scar was detected either as an electrically quiescent area or a line of double potentials, continuous activation was demonstrated around the scar and the flutter terminated during ablation between the scar and a barrier such as the inferior vena cava or the tricuspid ring (Fig. 2). A non-CTI-dependent AFL was defined as perimitral flutter if the mitral isthmus (between the left-sided pulmonary veins and the mitral annulus) proved to be in the circuit based on entrainment, activation proceeded around the mitral ring and the flutter terminated during ablation between the mitral annulus and another barrier (Fig. 3). In case of a roof-dependent LA flutter the activation proceeded around the left or right pulmonary veins (PVs) and/or an area of scar on the posterior LA wall, the LA roof proved to be in the circuit by entrainment and the flutter terminated during linear ablation between upper PVs or the mitral isthmus in case of peri-left PV AFL. Upper loop reentry was defined as a flutter circuit in the RA, around the ostium of the superior caval vein. 


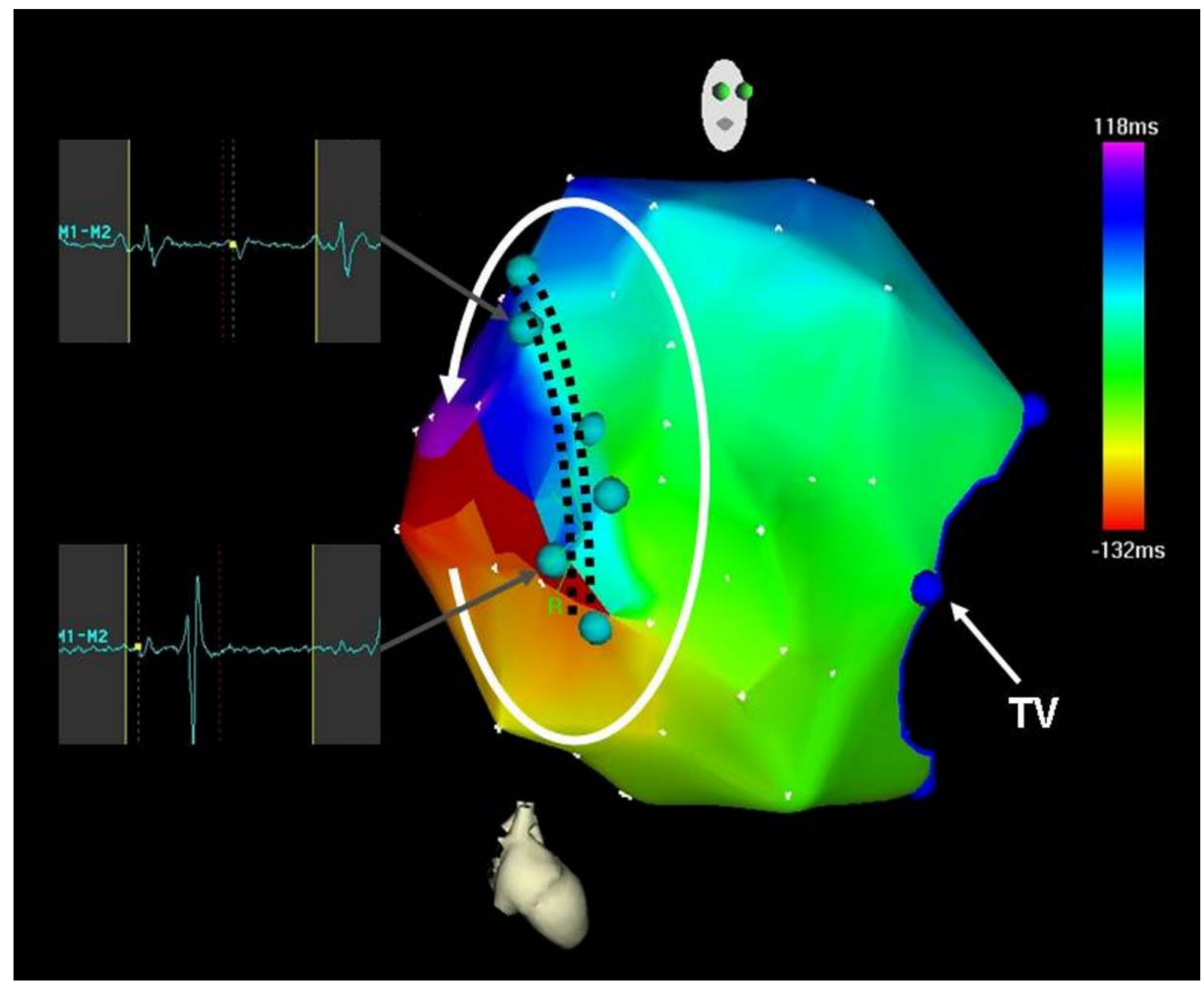

Figure 2. Electroanatomic activation map of the right atrium during incisional tachycardia after right lateral atriotomy. The atriotomy is marked by light blue dots, indicating double potentials (see inlets for examples of such potentials) and parallel dotted lines. Activation proceeds in the counterclockwise direction around the atriotomy. Dark blue dots mark the tricuspid valve (TV) 


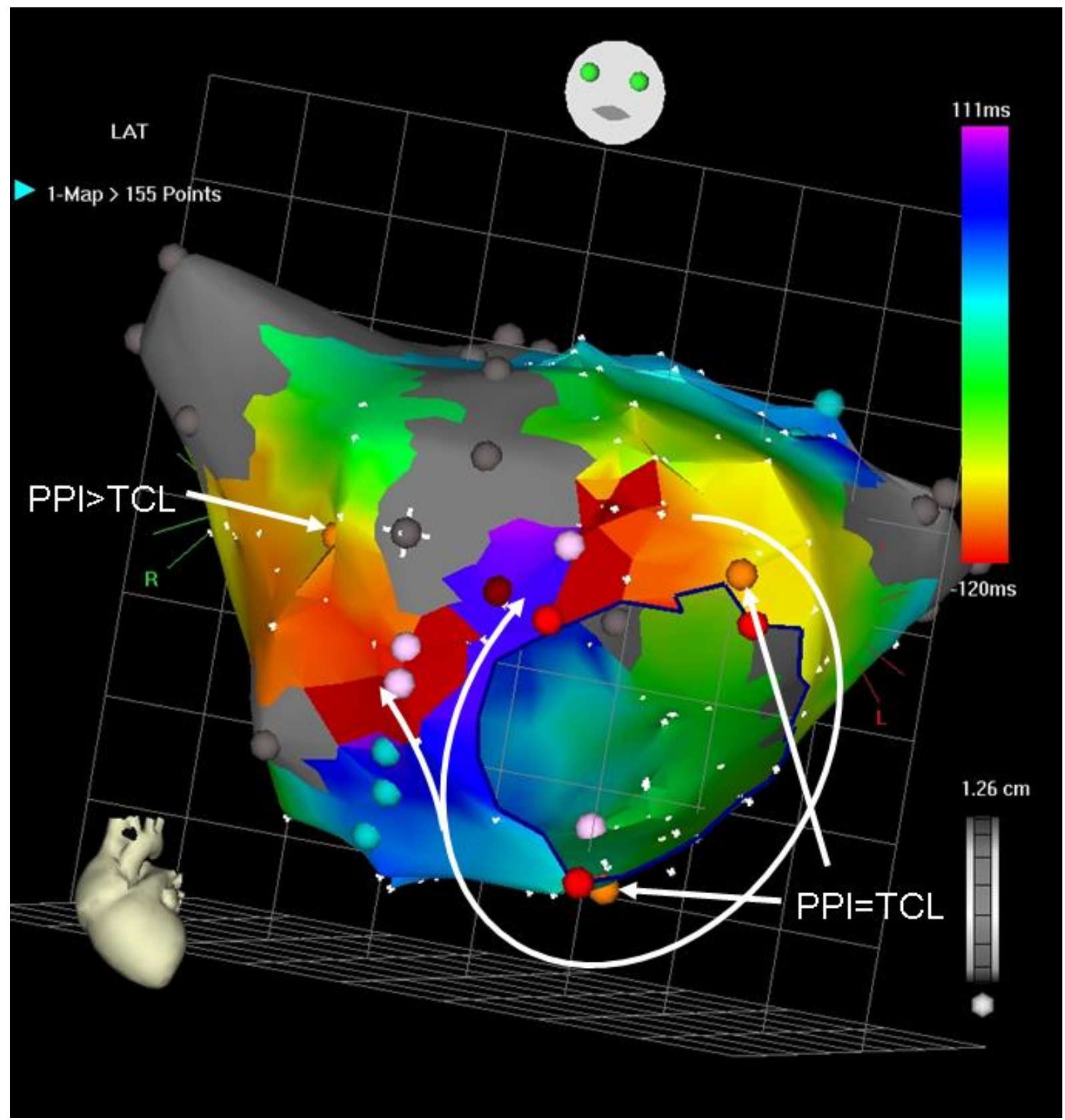

Figure 3. Perimitral macroreentry after direct left atriotomy. Activation proceeds in the clockwise direction around the mitral annulus and also through a reconnection in the atriotomy line. This latter path is not part of the circuit as indicated by entrainment pacing. The circuit uses a narrow isthmus between the scar and the mitral annulus, where a single radiofrequency pulse (dark red dot) terminated the tachycardia. Orange dots represent pacing sites, light blue dots mark double potentials, grey dots, electrically silent areas, and pink dots represent fragmented signals. Light red dots mark the mitral annulus. PPI post-pacing interval after entrainment, TCL tachycardia cycle length. 


\section{$\underline{\text { Statistical analysis }}$}

Continuous variables are presented as mean \pm SD and were compared using Student's $t$ test. Categorical variables are presented as frequencies. Comparisons of proportions were performed by the chi-square test. If more than one variable was significantly related to a specific AT mechanism multivariable analysis by logistic regression was performed. A P value $<0.05$ was considered statistically significant. Statistical analyses were performed using SPSS software, version 13.0 (SPSS Inc., Chicago, IL, USA).

\section{Results 1.}

\section{$\underline{\text { Baseline Clinical Characteristics }}$}

Baseline patient characteristics are shown in Table 3. The patients' mean age was $61.3 \pm 8.9$ years (range 36-78 years). This group of patients was predominantly male and hypertensive. Seventy-seven percent of patients had persistent AF, whereas $23 \%$ had longstanding persistent AF. All patients had preserved LVEF (> 50\%).

\section{Procedural Outcomes}

A total of 125 patients were included in the study. The mean follow-up time of the study population was $17.8 \pm 7.7$ months. After the first PVI, success was achieved in 73 patients (58.4\%). Twenty patients had a second PVI and 10 of these patients remained in sinus rhythm (SR) during follow-up. Three patients had a third PVI but all failed to maintain SR. At the end of the follow-up period, 83 patients remained in SR (66.4\%) and 42 (33.6\%) had AF recurrence. 


\begin{tabular}{|lc|}
\hline Characteristics & Total $(\mathbf{n}=\mathbf{1 2 5})$ \\
\hline Demographics and Comorbidities & \\
Age $($ years) & $61.3 \pm 8.9$ \\
BSA $\left(\mathrm{m}^{2}\right)$ & $2.1 \pm 0.3$ \\
Gender $(\%$ male) & 81 \\
Hypertension $(\%)$ & 66 \\
Diabetes mellitus (\%) & 15 \\
Stroke $(\%)$ & 9 \\
LVEF $(\%)$ & $60.7 \pm 6.4$ \\
Coronary artery disease $(\%)$ & 13 \\
Pacemaker or ICD $(\%)$ & 2 \\
Asthma, COPD $(\%)$ & 8 \\
Obstructive sleep apnea (\%) & 25 \\
Atrial Fibrillation & 23 \\
Longstanding persistent (\%) & 77 \\
Persistent (\%) & $12.7 \pm 20.2$ \\
Duration of AF (months) & \\
Baseline Medications (\%) & 26 \\
ACE inhibitor & 14 \\
Angiotensin receptor blocker & 28 \\
Diuretic & 2 \\
Inhaled $\beta$-agonist & 42 \\
$\beta$-blocker & 32 \\
AAD Class I & 68 \\
AAD Class III & 23 \\
AAD Class IV & 16 \\
Digoxin & $17.8 \pm 7.7$ \\
Mean Follow-Up Time (Months) & \\
&
\end{tabular}

Table 3. Baseline Characteristics of Patients, $\mathrm{AAD}=$ antiarrhythmic drug; $\mathrm{ACE}=$ angiotensin-converting enzyme; $\mathrm{AF}=$ atrial fibrillation; $\mathrm{BSA}=$ body surface area; $\mathrm{COPD}=$ chronic obstructive pulmonary disease; ICD =implantable cardioverter defibrillator; $\mathrm{LVEF}=$ left ventricular ejection fraction.

\section{Clinical Parameters Associated with Recurrence}

The clinical differences between patients with and without recurrences are summarized in Table 4. Patients with AF recurrences were more likely to have longer duration of AF prior to RFA $(9.0 \pm 9.4$ months vs. $19.3 \pm 30.6$ months, $\mathrm{P}=0.02)$. Although not statistically significant, patients who were free from recurrences were more likely to have lone AF 
(17\% vs. $7.2 \%, \mathrm{P}=0.13$ ). According to the logistic regression analysis, the duration of $\mathrm{AF}$ prior to RFA ( $\mathrm{P}=0.04$, odds ratio $[\mathrm{OR}]$ : $1.03,95 \%$ confidence interval $[\mathrm{CI}] 1.0-1.1)$ was statistically significant predictor for AF recurrence (Table 5.). The percentages of patients taking antiarrhythmic drugs were not different with respect to the outcome.

\begin{tabular}{|lccl|}
\hline & $\begin{array}{c}\text { Patients Without } \\
\text { Recurrence (N = 83) }\end{array}$ & $\begin{array}{c}\text { Patients with } \\
\text { Recurrence (N = 42) }\end{array}$ & P \\
\hline Clinical Parameters & & & \\
BSA $\left(\mathrm{m}^{2}\right)$ & $2.1 \pm 0.3$ & $2.2 \pm 0.3$ & 0.25 \\
Age & $60.7 \pm 8.5$ & $62.5 \pm 9.7$ & 0.16 \\
CHADS 2 score & $0.9 \pm 0.8$ & $1.1 \pm 0.9$ & 0.15 \\
Gender (male, $\%)$ & $69(83 \%)$ & $32(76 \%)$ & 0.35 \\
Hypertension & $52(63 \%)$ & $31(74 \%)$ & 0.21 \\
Diabetes mellitus & $13(16 \%)$ & $6(14 \%)$ & 0.8 \\
Coronary artery disease & $9(11 \%)$ & $7(17 \%)$ & 0.4 \\
COPD & $2(2 \%)$ & $2(5 \%)$ & 0.48 \\
Asthma & $2(2.5 \%)$ & $0(0 \%)$ & 0.31 \\
Obstructive sleep apnea & $18(22 \%)$ & $13(31 \%)$ & 0.3 \\
Lone AF & $14(17 \%)$ & $5(7.2 \%)$ & 0.13 \\
Longstanding persistent AF & $16(19 \%)$ & $13(31 \%)$ & 0.1 \\
Duration of AF prior to RFA (months) & $9.0 \pm 9.4$ & $19.3 \pm 30.6$ & 0.007 \\
Medications & & & \\
$\beta$-blocker & $57(71 \%)$ & $27(68 \%)$ & 0.67 \\
AAD Class I & $25(31 \%)$ & $10(25 \%)$ & 0.47 \\
AAD Class III & $24(30 \%)$ & $17(43 \%)$ & 0.17 \\
AAD Class IV & $17(21 \%)$ & $11(28 \%)$ & 0.45 \\
Digoxin & $6(8 \%)$ & $3(8 \%)$ & 1 \\
\hline
\end{tabular}

Table 4. Clinical Characteristics of the Patients with and Without Recurrence, CHADS$_{2}$ (congestive heart failure, hypertension, age $\geq 75$ years, diabetes mellitus, stroke or TIA or thromboembolism). The medications were taken by the patients at the end of the follow-up period. RFA = radiofrequency ablation. Abbreviations as in Table $\mathrm{I}$. $\mathrm{CI}=$ confidence interval; $\mathrm{OR}=$ odds ratio. 


\begin{tabular}{|lcc|}
\hline & \multicolumn{2}{c|}{$\begin{array}{c}\text { Univariate } \\
\text { Logistic Regression } \\
\text { OR [95\% CI] }\end{array}$} \\
\hline Clinical Parameters & P & \\
BSA $\left(\mathrm{m}^{2}\right)$ & 0.50 & $1.8[0.4-9.13]$ \\
Age & 0.30 & $1.0[0.94-1.06]$ \\
CHADS 2 score & 0.30 & $1.3[0.81-1.9]$ \\
Gender (male, \%) & 0.40 & $0.7[0.26-1.62]$ \\
Hypertension & 0.20 & $1.7[0.7-3.8]$ \\
Diabetes mellitus & 0.84 & $0.9[0.31-2.6]$ \\
Coronary artery disease & 0.36 & $1.6[0.6-4.8]$ \\
COPD & 0.50 & $2.0[0.28-14.9]$ \\
Obstructive sleep apnea & 0.26 & $1.6[0.7-3.7]$ \\
Lone AF & 0.14 & $0.4[0.1-1.4]$ \\
Longstanding persistent AF & 0.14 & $1.9[0.8-4.4]$ \\
Duration of AF prior RFA & 0.04 & $1.03[1.0-1.06]$ \\
Medication & & \\
AAD Class I & 0.50 & $0.7[0.3-1.72]$ \\
AAD Class III & 0.17 & $1.7[0.8-3.8]$ \\
AAD Class IV & 0.45 & $1.4[0.6-3.4]$ \\
$\beta$-blocker & 0.70 & $0.8[0.4-1.9]$ \\
Digoxin & 1.00 & $1.0[0.24-4.2]$ \\
\hline
\end{tabular}

Table 5. Logistic Regression Analysis on Clinical Parameters for Predicting Recurrence, Abbreviations as in Tables 3 and 4.

\section{Echocardiographic and Atrial Pressure Parameters}

Several echocardiographic parameters were found to be significantly different in patients with and without AF recurrence, as shown in Table 6. All the LA size measurement at the 4C view, including both maximum and minimum lengths, area, and volume measurements, were significantly correlated with outcome. None of the TDI parameters or direct measured LAP was significantly different. Among the 2D parameters, LAVimin of $26 \mathrm{~cm}^{3} / \mathrm{m}^{2}$ $\left(\mathrm{P}=0.033\right.$, OR 2.5, 95\% CI 1.08-5.9) and LAVimax of $42 \mathrm{~cm}^{3} / \mathrm{m}^{2}(\mathrm{P}=0.015$, OR 2.7, 95\% CI 1.2-5.95) were the best single parameters of AF recurrence after PVI. LAVimax value of greater than $40 \mathrm{~cm}^{3} / \mathrm{m}^{2}$ is considered severely enlarged according to the American Society of Echocardiography guidelines. ${ }^{32}$ When entered into multiple logistic regression analysis, LAVimin of $26 \mathrm{~cm}^{3} / \mathrm{m}^{2}$ ( $\mathrm{P}=0.009$, OR: 4.9, 95\% CI 1.5-16.2) and duration of AF prior to RFA ( $\mathrm{P}=0.05$, OR: $1.03,95 \%$ CI 0.99-1.07) were the independent parameters for AF recurrence. 


\begin{tabular}{|c|c|c|c|}
\hline & $\begin{array}{c}\text { Patients Without } \\
\text { Recurrence }(\mathbf{N}=\mathbf{8 3})\end{array}$ & $\begin{array}{c}\text { Patients with } \\
\text { Recurrence }(\mathrm{N}=\mathbf{4 2})\end{array}$ & $\begin{array}{c}\mathbf{P} \\
\text { Value } \\
\end{array}$ \\
\hline \multicolumn{4}{|c|}{ Echocardiographic Characteristics 2D } \\
\hline LA diameter $(\mathrm{mm})$ & $43.0 \pm 13.5$ & $41.5 \pm 14.7$ & 0.3 \\
\hline LA maximum area $\left(4 \mathrm{C}, \mathrm{cm}^{2}\right)$ & $23.4 \pm 4.9$ & $26.4 \pm 5.4$ & 0.002 \\
\hline LA minimum area $\left(4 \mathrm{C}, \mathrm{cm}^{2}\right)$ & $17.2 \pm 4.4$ & $19.6 \pm 4.8$ & 0.004 \\
\hline LA max. length $(4 \mathrm{C}, \mathrm{cm})$ & $5.2 \pm 0.6$ & $5.6 \pm 0.7$ & 0.001 \\
\hline LA min. length $(4 \mathrm{C}, \mathrm{cm})$ & $4.5 \pm 0.6$ & $4.8 \pm 0.6$ & 0.003 \\
\hline LA maximum area $\left(2 \mathrm{C}, \mathrm{cm}^{2}\right)$ & $23.8 \pm 4.4$ & $24.9 \pm 5.5$ & 0.12 \\
\hline LA minimum area $\left(2 \mathrm{C}, \mathrm{cm}^{2}\right)$ & $17.9 \pm 4.4$ & $19.0 \pm 4.9$ & 0.11 \\
\hline LA max. length $(2 \mathrm{C}, \mathrm{cm})$ & $5.3 \pm 0.7$ & $5.4 \pm 0.7$ & 0.21 \\
\hline LA min. length $(2 \mathrm{C}, \mathrm{cm})$ & $4.5 \pm 0.6$ & $4.6 \pm 0.7$ & 0.13 \\
\hline $\operatorname{LAVmax}\left(\mathrm{cm}^{3}\right)$ & $92.7 \pm 26.0$ & $107.3 \pm 31.2$ & 0.005 \\
\hline $\operatorname{LAVmin}\left(\mathrm{cm}^{3}\right)$ & $60.7 \pm 20.5$ & $71.9 \pm 26.4$ & 0.007 \\
\hline LAVimax $\left(\mathrm{cm}^{3} / \mathrm{m}^{2}\right)$ & $42.5 \pm 12.2$ & $49.04 \pm 14.9$ & 0.01 \\
\hline $\operatorname{LAVimin}\left(\mathrm{cm}^{3} / \mathrm{m}^{2}\right)$ & $27.8 \pm 9.6$ & $33.6 \pm 12.1$ & 0.005 \\
\hline LA ejection fraction $(\%)$ & $34.8 \pm 11.0$ & $33.6 \pm 13.1$ & 0.3 \\
\hline \multicolumn{4}{|l|}{ PWD } \\
\hline $\mathrm{E}(\mathrm{cm} / \mathrm{s})$ & $93 \pm 20$ & $94 \pm 25$ & 0.41 \\
\hline Deceleration time (seconds) & $198 \pm 42$ & $206 \pm 39$ & 0.2 \\
\hline PVS $(\mathrm{cm} / \mathrm{s})$ & $46 \pm 15$ & $45 \pm 17$ & 0.4 \\
\hline $\operatorname{PVD}(\mathrm{cm} / \mathrm{s})$ & $69 \pm 20$ & $62 \pm 16$ & 0.06 \\
\hline PVSD & $0.7 \pm 0.2$ & $0.8 \pm 0.3$ & 0.13 \\
\hline \multicolumn{4}{|l|}{ TDI } \\
\hline E' lateral $(\mathrm{cm})$ & $11.3 \pm 3.2$ & $10.8 \pm 2.4$ & 0.3 \\
\hline E' septal $(\mathrm{cm})$ & $9.5 \pm 2.9$ & $8.8 \pm 2.2$ & 0.08 \\
\hline $\mathrm{E}^{\prime}$ average $(\mathrm{cm})$ & $10.3 \pm 2.7$ & $9.8 \pm 2.1$ & 0.2 \\
\hline E/E' lateral & $9.2 \pm 3.9$ & $9.1 \pm 3.2$ & 0.5 \\
\hline E/E' septal & $10.7 \pm 3.9$ & $11.6 \pm 4.9$ & 0.15 \\
\hline E/E' average & $9.9 \pm 3.8$ & $10.1 \pm 3.7$ & 0.4 \\
\hline \multicolumn{4}{|l|}{ Atrial Pressure (mm Hg) } \\
\hline Mean RA pressure & $10.5 \pm 4.1$ & $10.7 \pm 4.2$ & 0.5 \\
\hline Mean LA pressure & $15.1 \pm 5.5$ & $16.1 \pm 5.9$ & 0.2 \\
\hline
\end{tabular}

Table 6. Invasive and Noninvasive Diastolic Function Parameters, $2 \mathrm{C}=2$-chamber view; $4 \mathrm{C}=4$-chamber view; $\mathrm{E}$ = early diastolic peak transmitral flow velocity; E' lateral, septal, avg = annular velocity at lateral, septal side, and the average of them, respectively; LA = left atrium; LAA = LA area; LAV = LA volume; LAVi = LA volume indexed to body surface area; $\mathrm{LAV} \max =\mathrm{LA}$ maximum volume; $\mathrm{LAVmin}=\mathrm{LA}$ minimum volume; 
$\mathrm{PVD}=$ pulmonary vein diastolic flow velocity; PVS = pulmonary vein systolic flow velocity;

$\mathrm{PVSD}=$ ratio of PVS and PVD; RA = right atrium.

\section{$\underline{\text { Reproducibility }}$}

Inter- and intraobserver variabilities for echocardiographic measurements are shown in Table 7. With the exception of the LA diameter measurements in the parasternal long axis view, intraobserver variability was minimal. Interobserver agreement was also excellent.

\begin{tabular}{|llccc|}
\hline Parameters & R & $\begin{array}{c}\text { Intraobserver } \\
\text { Difference (\%) }\end{array}$ & R & $\begin{array}{c}\text { Interobserver } \\
\text { Difference (\%) }\end{array}$ \\
\hline 2D & & & & \\
$\quad$ LA diameter & 0.7 & 4.5 & 0.6 & 1.3 \\
LA maximum area (4C) & 1.0 & 5.2 & 0.9 & 6.7 \\
LA maximum area (2C) & 0.9 & 2.5 & 0.8 & 0.4 \\
LAVmax & 0.9 & 0.8 & 0.8 & 2.4 \\
LAVmin & 0.8 & 1.5 & 0.8 & 7.7 \\
LAVimax & 0.9 & 2.7 & 0.8 & 7.0 \\
LAVimin & 0.8 & 1.2 & 0.8 & 8.3 \\
PWD & & & & \\
E & 0.9 & 2.5 & 1.0 & 1.4 \\
Deceleration time & 0.9 & 0.1 & 0.9 & 2.5 \\
PVS & 0.9 & 2.7 & 1.0 & 3.3 \\
PVD & 1.0 & 1.0 & 1.0 & 3.7 \\
PVSD & 0.9 & 1.1 & 0.7 & 1.2 \\
TDI & & & & \\
E' lateral & 1.0 & 2.6 & 1.0 & 3.6 \\
E' septal & 1.0 & 2.0 & 0.9 & 3.3 \\
E/E' lateral & 1.0 & 1.5 & 0.9 & 3.6 \\
E/E' septal & 1.0 & 0.5 & 0.9 & 2.7 \\
E/E' average & 1.0 & 1.0 & 0.9 & 2.9 \\
\hline
\end{tabular}

Table 7. Intra- and Interobserver Variability, Abbreviations as in Table 6.

\section{Results 2.}

\section{$\underline{\text { Patient characteristics }}$}

Of 1188 patients who underwent first time catheter ablation between 2004 and 2008, we identified 439 consecutive patients with AF recurrence after the blanking period with at least 18-month follow-up after recurrence. The first recurrence after catheter ablation occurred 
early (E group: 3-6 months after ablation) in 245 patients, late (L group: 6-12 months after ablation) in 118 patients, and very late (VL group: > 12 months after ablation) in 76 patients. Patient characteristics among the 3 groups categorized according to the time of recurrence after AF ablation were similar in terms of age, type of AF, prevalence of risk factors, and the presence of structural heart disease (Table 8). The mean follow-up after initial ablation was $50 \pm 18$ months (maximum 101 months), and the mean follow-up time after recurrence was $41 \pm 19$ months (maximum 97 months). 


\begin{tabular}{|c|c|c|c|c|}
\hline & Early group $(n=245)$ & Late group $(n=118)$ & $\begin{array}{l}\text { Very late group } \\
\quad(n=76)\end{array}$ & $\mathbf{P}$ \\
\hline Time to recurrence after ablation (mo) & $3.4 \pm 0.7$ & $9.3 \pm 2.2$ & $25.8 \pm 11.1$ & $<0.001$ \\
\hline Age $(y)$ & $57 \pm 10$ & $57 \pm 9$ & $58 \pm 10$ & 0.4 \\
\hline Sex: Man & $185(76)$ & $91(77)$ & $57(75)$ & 0.9 \\
\hline Body mass index $\left(\mathrm{kg} / \mathrm{m}^{2}\right)$ & $29.7 \pm 5.5$ & $28.9 \pm 4.9$ & $29.7 \pm 5.1$ & 0.5 \\
\hline Hypertension & $130(53)$ & $60(51)$ & $42(60)$ & 0.8 \\
\hline Diabetes mellitus & $17(7)$ & $11(9)$ & $8(11)$ & 0.5 \\
\hline Obstructive slee papnea & $44(18)$ & $18(15)$ & $15(21)$ & 0.7 \\
\hline Coronary artery disease & $33(13)$ & $12(10)$ & $11(15)$ & 0.6 \\
\hline Structural heart disease & $34(14)$ & $9(8)$ & $7(9)$ & 0.2 \\
\hline Left atrial size (mm) & $45 \pm 7$ & $44 \pm 7$ & $45 \pm 7$ & 0.7 \\
\hline Left ventricular ejection fraction (\%) & $57 \pm 9$ & $58 \pm 10$ & $59 \pm 9$ & 0.7 \\
\hline Paroxysmal AF & $144(59)$ & $67(57)$ & $42(60)$ & 0.8 \\
\hline AF duration $(y)$ & $6.9 \pm 6.5$ & $6.5 \pm 5.5$ & $5.4 \pm 4.2$ & 0.1 \\
\hline Rare AF episodes prior to ablation & $44(18)$ & $13(11)$ & $10(13)$ & 0.4 \\
\hline Number of failed AADs & $1.6 \pm 0.9$ & $1.5 \pm 0.9$ & $1.3 \pm 0.8$ & 0.1 \\
\hline Patients never on any AAD prior to ablation & $25(10)$ & $13(11)$ & $10(13)$ & 0.3 \\
\hline Tachycardia-mediated CM & $24(10)$ & $12(10)$ & $9(13)$ & 0.9 \\
\hline
\end{tabular}

Table 8. Baseline patient characteristics based on the time of AF recurrence after ablation, Continuous variables are expressed as mean \pm SD and categorical variables as number (percentages). $\mathrm{AAD}=$ antiarrhythmic drug; $\mathrm{AF}=$ atrial fibrillation; $\mathrm{CM}=$ cardiomyopathy. 


\section{Recurrences during the 3-month blanking period}

During the blanking period, 306 of 439 (70\%) of the study patients had arrhythmia episodes. Patients with early recurrences (3-6 months after ablation) were more likely to have had AF during the blanking period compared with patients with late and very late recurrences (201 of $245(82 \%)$ in the E group vs. 64 of $118(54 \%)$ in the L group and 41 of $76(54 \%)$ in the VL group; $\mathrm{P}<0.001$ for comparison across groups). DCCV was performed during the blanking period in 79 of 201 (39\%) in the E group, 28 of 64 (40\%) in the L group, and 22 of 41 (54\%) in the VL group $(\mathrm{P}=0.2)$. In total, 19 patients underwent a repeat ablation procedure during the blanking period, with no statistically significant difference between groups (13 of 201 $(6 \%)$ in the E group, 3 of $64(5 \%)$ in the L group, and 3 of $41(7 \%)$ in the VL group; $(\mathrm{P}=0.8)$.

\section{$\underline{\text { AF recurrences after the blanking period }}$}

At the time of recurrence after the blanking period, 144 of 245 (59\%) patients in the E group were on an AAD in comparison to 37 of 118 (31\%) patients in the L group and 5 of $76(7 \%)$ patients in the VL group $(\mathrm{P}<0.001)$. To treat recurrences, a total of 159 patients underwent at least $1 \mathrm{DCCV}$, without statistically significant differences among the 3 groups (81 of 245 [33\%] in the E group, 45 of 118 [38\%] in the L group, and 33 of 76 [43\%] in the VL group; $\mathrm{P}=0.2)$. Forty-two of 439 patients $(9.5 \%)$ recurred with atrial flutter or atrial tachycardia, with no statistical differences among the groups (30 of 245 [12\%] in the E group, 7 of 118 [6\%] in the L group, and 5 of 76 [7\%] in the VL group; $\mathrm{P}=0.1$ ). The presence of only subsequent rare AF recurrences was significantly higher in L and VL groups than in the E group (Figure 4.). Only 23 of 245 [9\%] patients in the E group had no or rare $\mathrm{AF}$ ( $\leq 2$ episodes or $\leq 1 \mathrm{DCCV}$ during any 6-month window from the first recurrence) in comparison to 50 of 118 (47\%) patients in the L group and 52 of $76(68 \%)$ in the VL group ( $\mathrm{P}<0.001$ for comparison across groups and for comparison between L and VL groups). There was no significant difference in the subsequent frequency of rare AF between those whose initial recurrence required DCCV and those whose initial recurrence did not require DCCV (39 of 141 [27.7\%] vs. 86 of 298 [28.9\%], respectively; $\mathrm{P}=0.8$ ). 


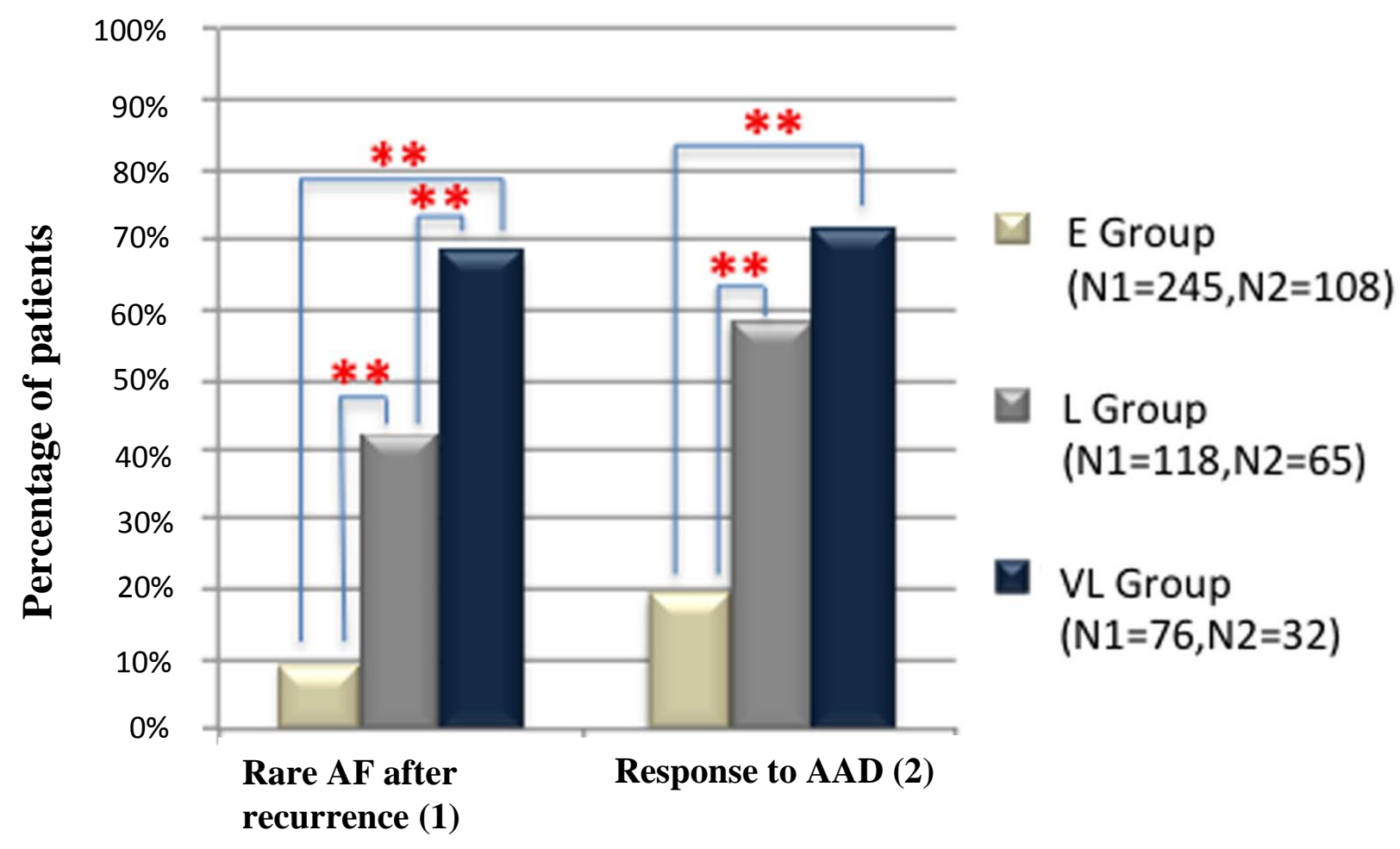

Figure 4. Influence of early (E), late (L), and very late (VL) recurrences after catheter ablation on the presence of no or only rare atrial fibrillation (AF) during follow-up and response to antiarrhythmic drugs (AADs) among all patients, ${ }^{*} \mathrm{P}<0.05 ; * * \mathrm{P}<0.001$.

Univariate factors predictive of rare arrhythmia episodes after recurrence are depicted in Table 9. Type of AF and patient clinical characteristics did not predict subsequent rare arrhythmia episodes after the initial AF recurrence. In multivariate analysis, the strongest independent predictors of rare AF after the initial AF recurrence were late and very late time of recurrence after the procedure. 


\begin{tabular}{|c|c|c|c|c|c|c|}
\hline & \multicolumn{3}{|c|}{ Univariate } & \multicolumn{3}{|c|}{ Multivariate } \\
\hline & OR & $95 \% \mathrm{CI}$ & $\mathbf{P}$ & OR & $95 \% \mathrm{CI}$ & $\mathbf{P}$ \\
\hline $\mathrm{AF}$ in the blanking period & 0.3 & $0.2-0.4$ & $<0.001$ & 0.5 & $0.3-0.9$ & 0.03 \\
\hline DCCV in the blanking period & 0.6 & $0.4-1.1$ & 0.06 & 0.93 & $0.5-2$ & 0.9 \\
\hline On $\mathrm{AAD}$ at the time of recurrence & 0.2 & $0.1-0.3$ & $<0.001$ & 0.5 & $0.3-0.9$ & 0.03 \\
\hline $\mathrm{L}$ recurrence group* & 7.09 & $4.03-12.5$ & $<0.001$ & 5.07 & $2.8-9.2$ & $<0.001$ \\
\hline VL recurrence group* & 20.8 & $10.9-40$ & $<0.001$ & 13.8 & $6.9-27.7$ & $<0.001$ \\
\hline
\end{tabular}

Table 9. Factors predictive of rare $\mathrm{AF}$ after the initial recurrence, $\mathrm{AAD}=$ antiarrhythmic drug; $\mathrm{AF}=$ atrialfibrillation; $\mathrm{CI}=\mathrm{confidence}$ interval; $\mathrm{DCCV}=$ direct current cardioversion; $\mathrm{L}=$ late $(6-12$ mo after ablation); $\mathrm{VL}=$ very late $(>12$ mo after ablation). *Relative to the $\mathrm{E}$ recurrence group, OR= Odds Ratio 


\section{Efficacy of antiarrhythmic drug therapy after recurrence}

AADs were tried in 108 of 245 (44\%), 65 of 118 (55\%), and 32 of 76 (42\%) patients in the E, $\mathrm{L}$, and VL recurrence groups, respectively $(\mathrm{P}=0.1$ for comparison across groups). Seventytwo patients were started on a new AAD despite having rare arrhythmia episodes before AAD initiation (17 of 108 [16\%] in the E group, 38 of 65 [58\%] in the L group, and 17 of 32 [53\%] in the VL group; $\mathrm{P}<0.001)$. There were no differences in the type of drug used among the groups; class III AADs were the most frequently selected (49\%), followed by class I AADs $(33 \%)$ and amiodarone (13\%). Patients in the VL group were more likely to respond to AADs with the elimination of AF during subsequent follow-up than patients with early and late recurrences (23 of 32 [72\%] showed positive response in the VL group vs. 21 of 108 [19\%] in the E group and 38 of 65 [58\%] in the L group; $\mathrm{P}<0.001$ ) (Figure 1A). The difference was maintained regardless of the frequency of episodes prior to initiating AADs (8 of 15 [53\%] showed positive response in the VL group vs. 13 of 91 [14\%] in the E group and 9 of 27 [33\%] in the $\mathrm{L}$ group; $\mathrm{P}=0.001)$. Patients with paroxysmal $\mathrm{AF}$, those who did not recur during the blanking period, those not on AADs at the time of recurrence, and those with rare AF episodes after recurrence had a better response to AADs. The class of AADs did not impact positive response ( $44 \%$ class III, $38 \%$ class I, $32 \%$ amiodarone; $\mathrm{P}=0.6$ ), nor did other clinical baseline characteristics. In a multivariate model, baseline paroxysmal AF, the time to AF recurrence after ablation, and rare AF prior to initiating or changing AADs were independent predictors of a positive response to AAD therapy after the initial recurrence (Table 10).

\begin{tabular}{|lcccccc|}
\hline & \multicolumn{3}{c}{ Univariate } & \multicolumn{3}{c|}{ Multivariate } \\
& OR & $\mathbf{9 5 \%}$ CI & P & OR & $\mathbf{9 5 \%}$ CI & P \\
\hline Paroxysmal AF & 1.7 & $0.9-3.05$ & 0.06 & 2.2 & $1.05-4.6$ & 0.04 \\
AF in the blanking period & 0.3 & $0.2-0.5$ & $<0.001$ & 0.6 & $0.2-1.4$ & 0.2 \\
CV of AF in the blanking period & 0.5 & $0.3-0.9$ & 0.04 & 0.7 & $0.3-1.6$ & 0.4 \\
On AAD at the time of & & & & & & \\
recurrence & 0.3 & $0.1-0.6$ & 0.001 & 0.8 & $0.3-2$ & 0.7 \\
Rare AF before ADD & 8.9 & $4.6-17.2$ & $<0.001$ & 5.4 & $2.5-11.7$ & $<0.001$ \\
L recurrence group* & 5.8 & $2.9-11.6$ & $<0.001$ & 3.1 & $1.4-6.8$ & 0.004 \\
VL recurrence group* & 10.5 & $4.3-26.2$ & $<0.001$ & 6.7 & $2.4-18.9$ & $<0.001$ \\
\hline
\end{tabular}

Table 10. Factors predictive of no $\mathrm{AF}$ on $\mathrm{AADs}$ after recurrence, $\mathrm{AAD}=$ antiarrhythmic drug; $\mathrm{AF}=$ atrial fibrillation; $\mathrm{CI}=$ confidence interval; $\mathrm{DCCV}=$ direct current cardioversion; $\mathrm{L}=$ late (6-12 mo after ablation); $\mathrm{VL}=$ very late $(>12$ mo after ablation). *Relative to the $\mathrm{E}$ recurrence group, $\mathrm{OR}=$ Odds ratio 


\section{$\underline{\text { Repeat ablation and outcome }}$}

In this study cohort, 290 patients underwent repeat ablation (185 of 245 [75\%] in the E group, 70 of 118 [59\%] in the L group, and 35 of 76 [46\%] in the VL group; P < 0.001) (Figure 5.). Patients in the E group were the most likely to pursue repeat ablation owing to frequent symptomatic recurrences (79\% in the E group vs. $47 \%$ in the L group and $29 \%$ in the VL group; $\mathrm{P}<0.001$ ). In patients with recurrences after 6 months, the decision to pursue repeat ablation was more often driven by patient preference for ablation over continued AADs (31\% in the VL group and $28 \%$ in the L group vs. $10 \%$ in the $\mathrm{E}$ group; $\mathrm{P}=0.001$ ), by the need for cardioversion or by the persistent nature of the AF recurrence (31\% in the VL group and $24 \%$ in the $\mathrm{L}$ group vs. $13 \%$ in the $\mathrm{E}$ group; $\mathrm{P}=0.01$ ). Two patients in each group underwent a repeat procedure because of tachycardia-induced cardiomyopathy, tachycardia-bradycardia syndrome, or episodes of heart failure associated with AF. After the second procedure, 241 patients had at least 1-year follow-up. Patients in the VL group had the highest rate of AF control (no or rare episodes for each year of follow-up) after repeat ablation (24 of 27 [89\%] vs. 38 of 54 [72\%] in the L group and 78 of 160 [49\%] in the E group; P < 0.001) (Figure 5). Other factors predictive of AF control after repeat AF ablation in univariate analysis included paroxysmal AF, LA size, the duration of AF, recurrences in the blanking period, and the presence of rare AF after recurrence. In multivariate analysis, the type of AF, LA size, the duration of AF history prior to ablation, and the time of recurrence after ablation independently predicted AF control after repeat ablation (Table 11). 


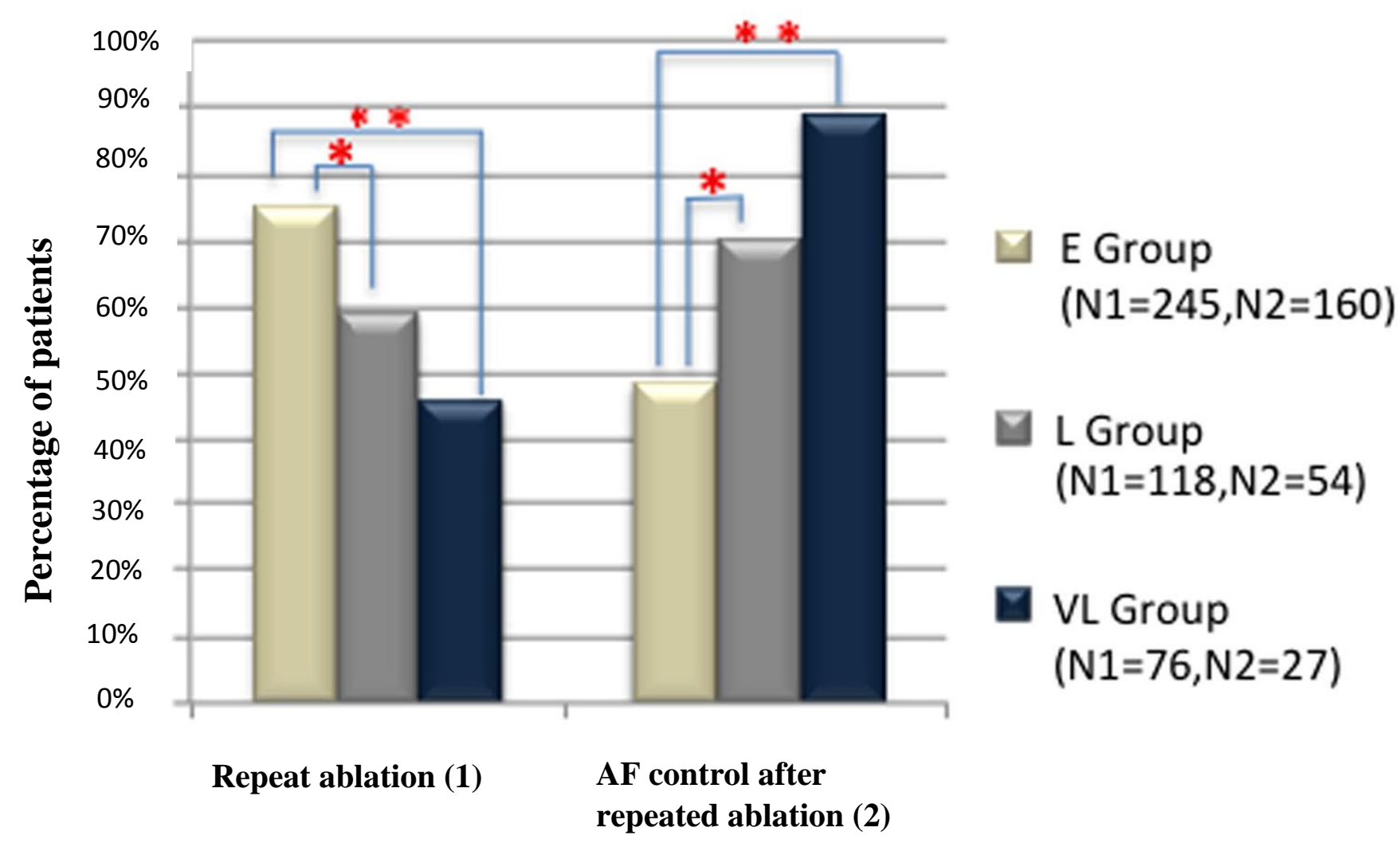

Figure 5. Influence of early (E), late (L), and very late (VL) recurrences after catheter ablation on the rate of repeat ablation and > 1-year atrial fibrillation (AF) control after repeat ablation in all patients. $* \mathrm{P}<0.05 ; * * \mathrm{P}<0.001$

\begin{tabular}{|lcccccc|}
\hline & \multicolumn{3}{c}{ Univariate } & \multicolumn{3}{c|}{ Multivariate } \\
& OR & $\mathbf{9 5 \%}$ CI & P & OR & 95\% CI & P \\
\hline LA size (mm) & 0.9 & $0.92-0.99$ & 0.03 & 0.9 & $0.9-0.99$ & 0.03 \\
Paroxysmal AF & 2.08 & $1.2-3.5$ & 0.006 & 2.5 & $1.2-4.9$ & 0.009 \\
AF duration before ablation (y) & 0.9 & $0.9-0.98$ & 0.003 & 0.9 & $0.9-0.98$ & 0.009 \\
AF in the blanking period & 0.5 & $0.3-0.9$ & 0.02 & 0.7 & $0.3-1.7$ & 0.3 \\
Rare AF after recurrence & 2.8 & $1.3-5.9$ & 0.008 & 1.5 & $0.5-4.5$ & 0.4 \\
L recurrence group* & 2.5 & $1.3-4.8$ & 0.007 & 1.7 & $0.7-3.9$ & 0.2 \\
VL recurrence group* & 8.4 & $2.4-29.05$ & 0.001 & 7.4 & $1.5-36.9$ & 0.014 \\
\hline
\end{tabular}

Table 11. Factors predictive of AF control after repeat ablation procedure, $\mathrm{AAD}=$ antiarrhythmic drugs; $\mathrm{AF}=$ atrial fibrillation; $\mathrm{CI}=$ confidence interval $; \mathrm{LA}=$ left atrium; $\mathrm{L}=$ late $(6-12 \mathrm{mo}$ after ablation); $\mathrm{VL}=$ very late $(>12 \mathrm{mo}$ after ablation). *Relative to the $\mathrm{E}$ recurrence group. $\mathrm{OR}=$ Odds ratio 


\section{Outcome of patients without repeat $\mathrm{AF}$ ablation}

The 149 patients who did not undergo a repeat ablation had a clinical course that varied based on the time of their first AF recurrence after the original ablation (Figure 6). Patients with very late recurrences did extremely well. Of the 41 patients in the VL group who did not undergo repeat ablation, 15 were free of $\mathrm{AF}$ after the initial recurrence and 24 experienced only rare subsequent AF recurrences (39 of 41 [95\%] patients with good outcomes, with a mean maximum arrhythmia-free interval of $21 \pm 7$ months). Of the 48 patients in the $\mathrm{L}$ recurrence group, 10 were free of $\mathrm{AF}$ and 27 patients had rare AF recurrences (37 of 48 [77\%] patients with good clinical outcome with a mean maximum arrhythmia-free interval of $16 \pm 5$ months). In contrast, of the 60 patients in the $\mathrm{E}$ group who did not undergo repeat ablation, only 3 patients remained arrhythmia-free and 19 had rare AF episodes after the first recurrence (22 of 60 [37\%] patients with good outcomes, with a mean maximum arrhythmiafree interval of $14.9 \pm 3.9$ months; $\mathrm{P}<0.001$ vs. L and VL groups). In patients who continued to have frequent episodes, 10 patients were treated with rate control, 1 underwent atrioventricular junction ablation, and 9 patients had been scheduled for a repeat ablation procedure after the end of the follow-up. Thirty patients had more than 2 episodes per 6 month follow-up window or $>1$ cardioversion but declined a repeat AF ablation procedure. Patients not undergoing repeat ablation with favorable outcome were less likely to have AF recurrence in the blanking period, less frequently on $\mathrm{AAD}$ at the time of $\mathrm{AF}$ recurrence after the blanking period, and had rare episodes in the first 6 months after recurrence. In multivariate analysis, the time of recurrence after ablation was the only independent predictor of no or rare arrhythmia episodes and patients in L and VL groups had more favorable outcome than did patients in the E recurrence group (Table 12). 


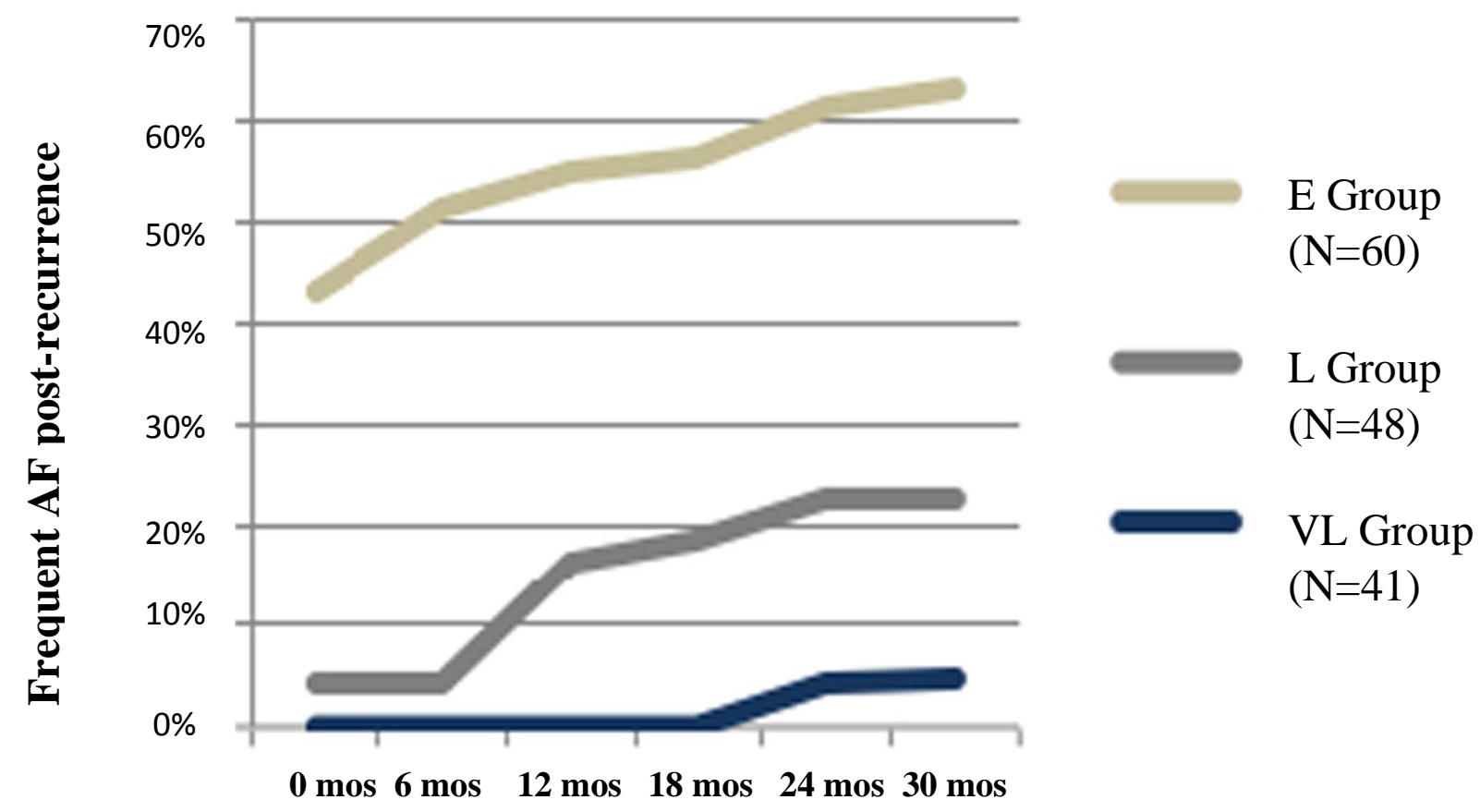

Figure 6. Influence of early (E), late (L), and very late (VL) recurrences after catheter ablation on the development of more frequent atrial fibrillation (AF), defined as $>2 \mathrm{AF}$ episodes or $>1$ cardioversion in any 6-month follow-up window. Included are all patients without repeat ablation and at least 30-month follow-up.

\begin{tabular}{|lcccccc|}
\hline & \multicolumn{3}{c}{ Univariate } & \multicolumn{3}{c|}{ Multivariate } \\
& OR & $\mathbf{9 5 \%}$ CI & P & OR & $\mathbf{9 5 \%}$ CI & P \\
\hline AF in the blanking period & 0.2 & $0.08-0.5$ & $<0.001$ & 0.4 & $0.1-1.1$ & 0.06 \\
Rare AF in the first 6 mo after first & & & & & & \\
recurrence & 4.5 & $2.2-9.4$ & $<0.001$ & 1.2 & $0.4-3.2$ & 0.7 \\
On AAD at the time of recurrence & 0.3 & $0.1-0.6$ & 0.002 & 0.8 & $0.3-2.4$ & 0.9 \\
L recurrence group* & 5.8 & $2.4-13.7$ & $<0.001$ & 3.9 & $1.5-10.2$ & 0.004 \\
VL recurrence group* & 33.3 & $7.4-142.8$ & $<0.001$ & 23.8 & $4.6-125$ & $<0.001$ \\
\hline
\end{tabular}

Table 12. Factors predictive of no or rare AF without repeat ablation after recurrence, $\mathrm{AAD}=$ antiarrhythmic drugs; $\mathrm{AF}=$ atrial fibrillation; $\mathrm{CI}=$ confidence interval; $\mathrm{L}=$ late $(6-12$ mo after ablation); $\mathrm{VL}=$ very late $(>12 \mathrm{mo}$ after ablation). *Relative to the $\mathrm{E}$ recurrence group, $\mathrm{OR}=$ Odds ratio

\section{Outcomes in paroxysmal and nonparoxysmal AF}

Patients with both paroxysmal and persistent AF demonstrated the same influence of the time of AF recurrence after ablation on clinical outcome (Figure 7, Figure 8, Figure 9). 

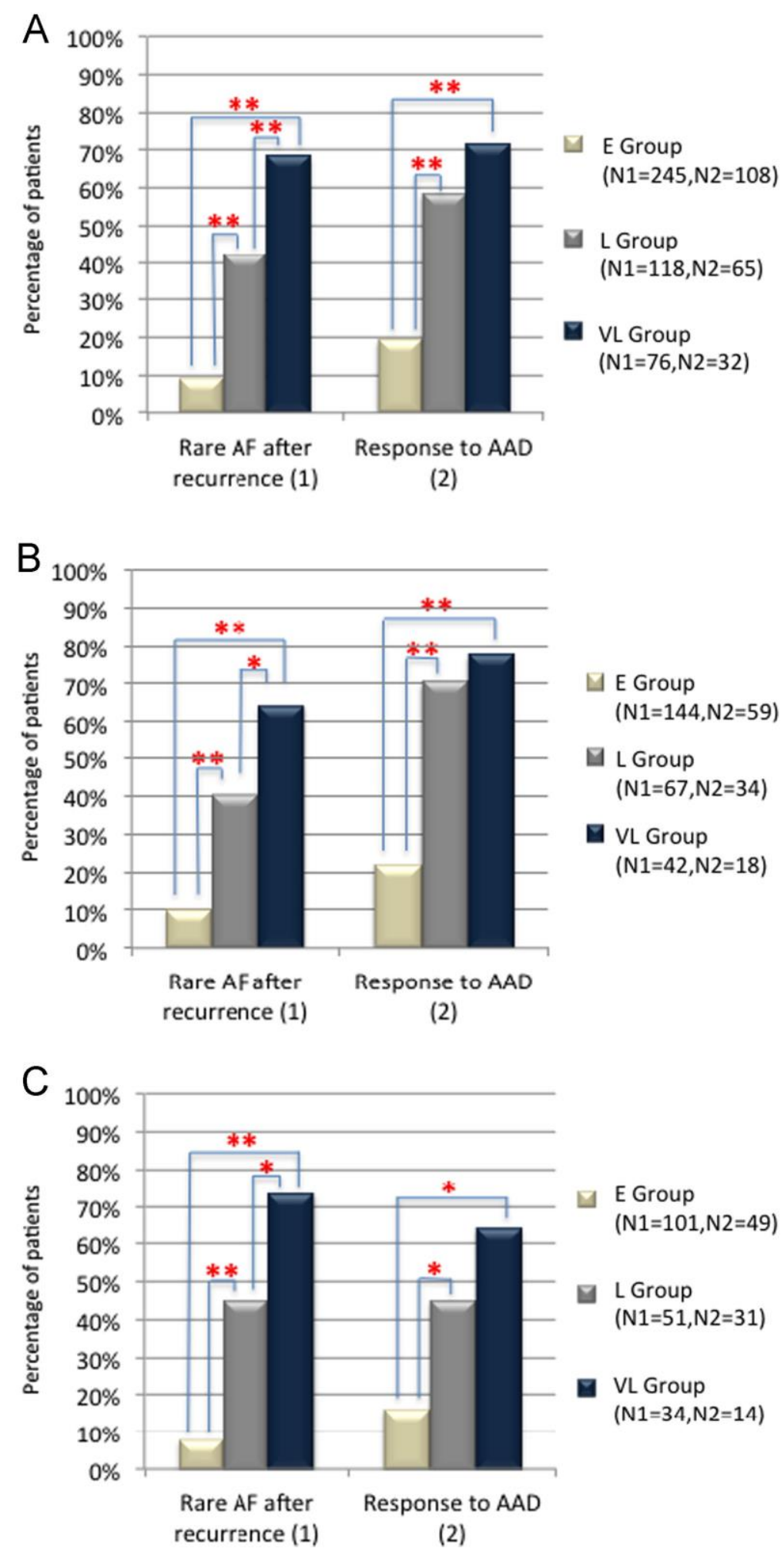

Figure 7. Influence of early (E), late (L), and very late (VL) recurrences after catheter ablation on the presence of no or only rare atrial fibrillation (AF) during follow-up and response to antiarrhythmic drugs (AADs) among all patients (A). Mean follow-up was 41 months. A similar response was observed among patients with paroxysmal (B) and persistent $\mathrm{AF}(\mathrm{C}) . * \mathrm{P}<0.05 ; * * \mathrm{P}<0.001$. 


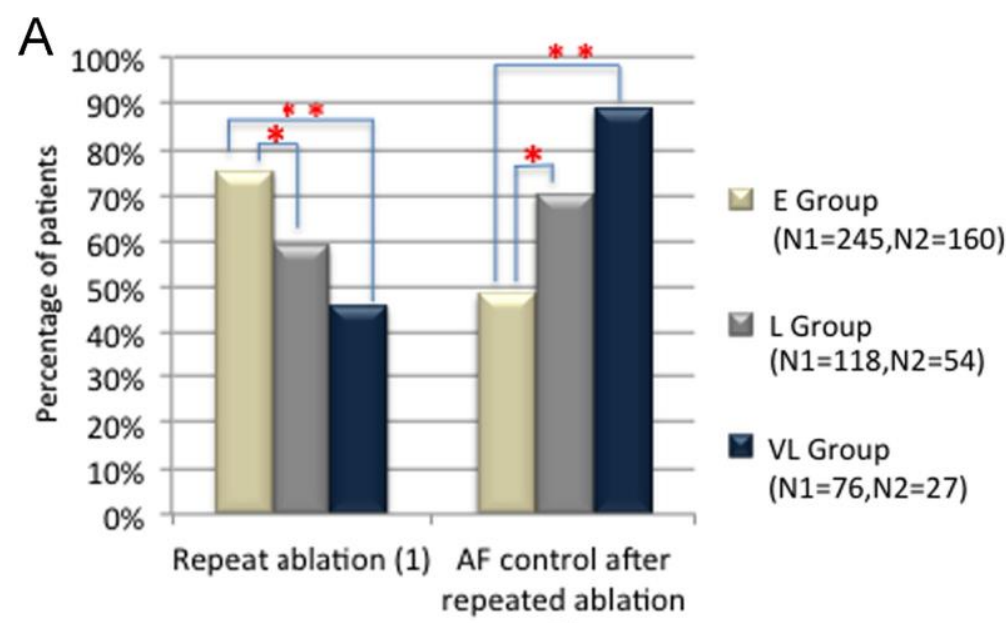

(2)

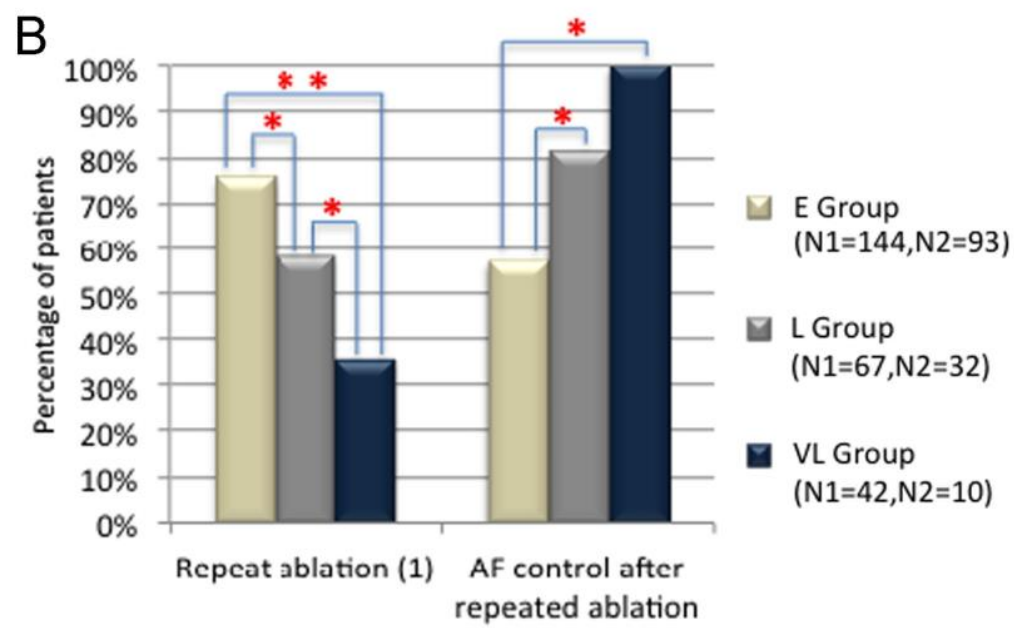

(2)

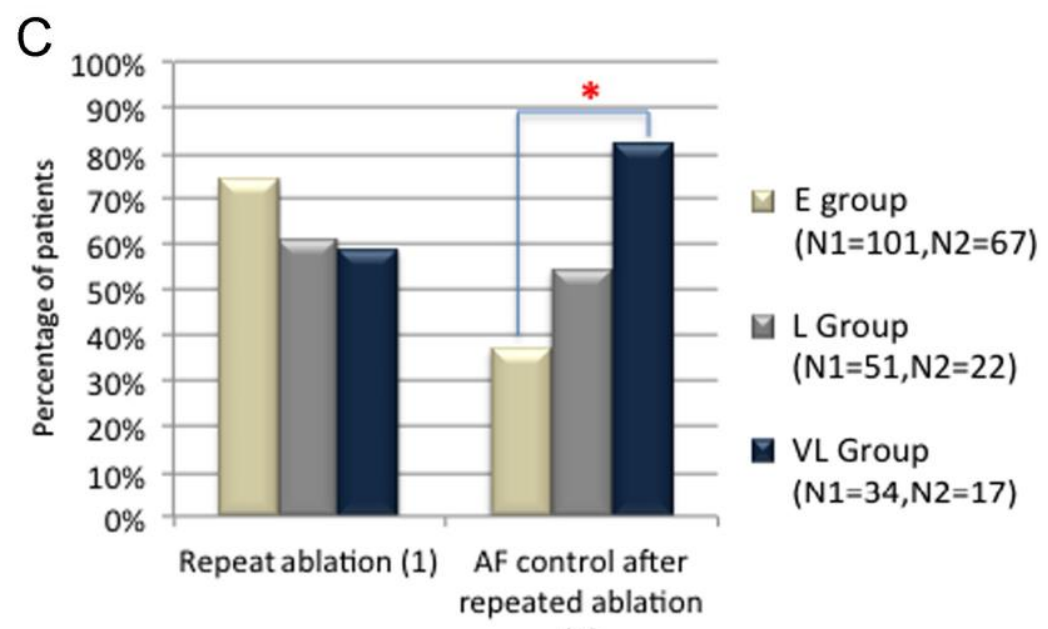

(2)

Figure 8. Influence of early (E), late (L), and very late (VL) recurrences after catheter ablation on the rate of repeat ablation and $1>$ year atrial fibrillation (AF) control after repeat ablation. A: All patients. B: Patients with paroxysmal AF. C: Patients with nonparoxysmal AF. $* \mathrm{P}<0.05 ; * * \mathrm{P}<0.001$. 

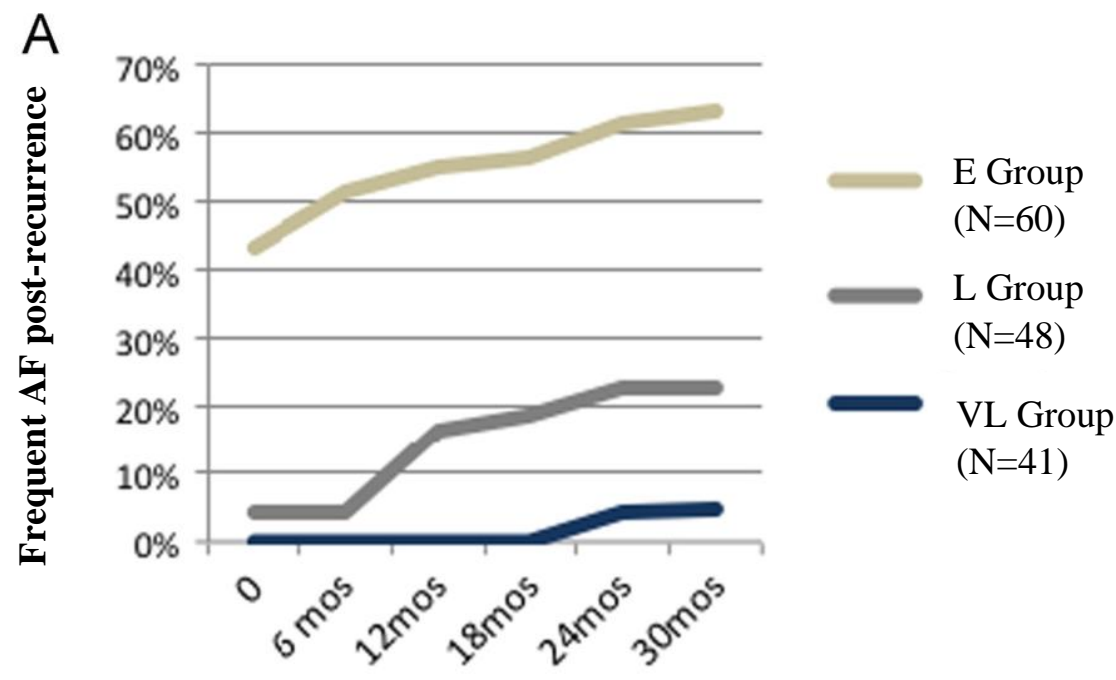

$\mathrm{B}$
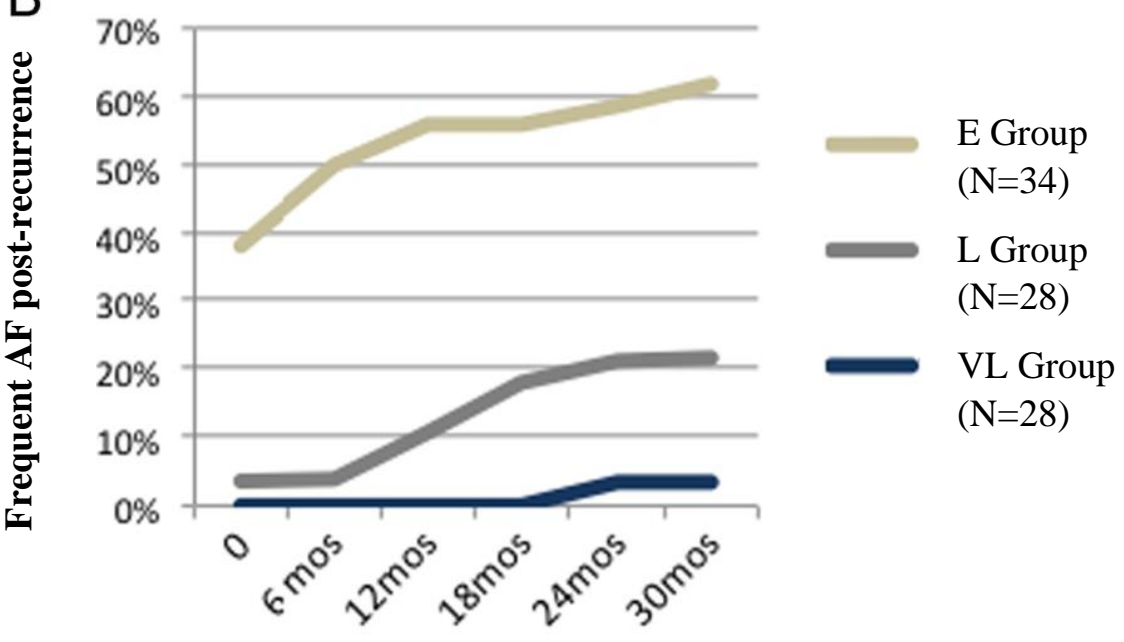

C

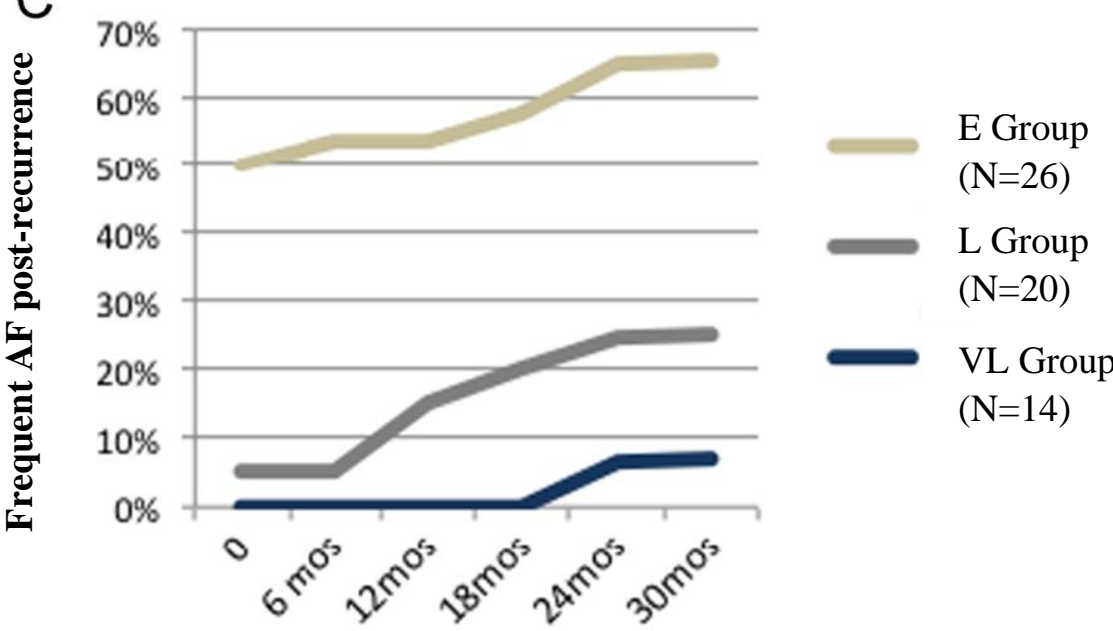

Figure 9. Influence of early (E), late (L), and very late (VL) recurrences after catheter ablation on the development of more frequent atrial fibrillation (AF), defined as $>2 \mathrm{AF}$ episodes or $>1$ cardioversion in any 6-month follow-up window. Included are all patients without repeat ablation and at least 30-month follow-up (A). In contrast to patients with early 
recurrences, patients with late recurrences typically had good outcome with no or infrequent AF during long-term follow-up. Results were similar for patients with paroxysmal (B) and persistent $\mathrm{AF}(\mathrm{C})$.

\section{Results 3.}

\section{Mechanism of postoperative atrial tachycardias}

One hundred patients had 151 atrial tachycardias (ATs) during 127 procedures-88 (58\%) CTI-dependent flutters, 34 (22.5\%) RA incisional tachycardias, 12 (8\%) perimitral flutters, 4 (3\%) LA roof dependent flutters, 1 (0.7\%) upper loop reentry, an unmapped LA flutter (failed transseptal access), and 11 (7\%) focal ATs. Eighty-eight patients had CTI-dependent flutter, and 49 patients had at least one non-CTI-dependent flutter. Eleven patients had focal atrial tachycardia. Focal ATs originated from the vicinity of surgical scar in 4 (36\%) of 11 cases (three from the RA free wall atriotomy, one from a septal ASD patch). Other origins of focal AT were at the LA roof, the pulmonary veins and the mitral ring in two (18\%) patients each and the tricuspid ring in one (9\%). In the case of 15 patients, atrial fibrillation was also observed during the procedure. The CARTO ${ }^{\mathrm{TM}}$ XP system was used for mapping in 69 procedures. Most CTI-dependent AFLs (64\%) were ongoing at the commencement of the procedure, while $18 \%$ occurred by transformation during ablation of another AT and $18 \%$ were induced by extrastimulation during sinus rhythm. The proportions of ongoing/ transformed/ induced ATs were $41 \%$ / 35\%/ 24\% of RA incisional, 67\%/ 17\%/ 17\% of perimitral, $50 \% / 25 \% / 25 \%$ of roof dependent flutters, and $18 \% / 46 \% / 36 \%$ of focal tachycardias.

\section{Distribution of atrial tachycardias between surgical groups}

Of the 20 patients with RA appendage cannulation, 19 (95\%) had CTI-dependent flutter, three (15\%) had a non-CTI-dependent AFL, and none had focal AT. All non-CTI-dependent AFLs in this group were RA incisional tachycardias related to the cannulation site of the RA. In patients with RA free wall atriotomy, a CTI-dependent AFL was seen in 30 of 32 (94\%) patients, a non-CTI-dependent AFL in 13 (41\%) and focal AT in four (13\%). Eleven of the 13 (85\%) non-CTI-dependent AFLs were RA incisional tachycardias related to the right atriotomy; there was one (8\%) perimitral AFL and one upper loop reentry in this group. Among patients with transseptal LA atriotomy the frequency of CTI-dependent flutter was $83 \%$ (34 of 41 patients), while at least one non-CTI-dependent AFL was seen in 30 patients 
(73\%). One patient (2\%) had two non-CTI-dependent circuits. Focal AT occurred in six (15\%) patients. The most frequently encountered non-CTIdependent AFL was again RA incisional tachycardia-20 of 31 circuits (65\%), while seven (23\%) perimitral and three (10\%) LA roof-dependent AFLs were mapped in this group. One LA AFL was not mapped due to failed transseptal access. Five of seven (71\%) patients had CTI-dependent AFL in the direct LA atriotomy group and four patients (57\%) had non-CTI-dependent AFLs. One patient (14\%) had two non-CTI-dependent circuits and one had focal AT. Perimitral was the most common non-CTI-dependent AFL-4 of 5 (80\%) circuits, while one (20\%) LA roofdependent AFL was diagnosed in this group.

\section{Comparison of AT mechanisms between surgical groups}

The frequency of CTI-dependent AFL was not different between groups ( $\mathrm{P}=0.195)$. A nonCTI-dependent AFL, on the other hand, was seen progressively more frequently with more extensive atrial incisions $(\mathrm{P}<0.001)-15 \%$ of patients who had simple venous cannula insertion, $41 \%$ and $57 \%$ after RA and LA atriotomy, respectively, and $73 \%$ of patients after biatrial access (transseptal left atriotomy). After operations involving the RA, the most common nonCTI-dependent circuit was that of a RA incisional tachycardia, seen more commonly in patients who had RA atriotomy as opposed to those who had only RA appendage cannulation ( $43 \%$ vs. $15 \%, \mathrm{P}=0.024$ ). Perimitral AFL was seen more frequently in cases where the LA was opened compared with operations involving only the RA-22\% versus $2 \%$, respectively $(\mathrm{P}=0.002)$, and the same was true for LA roof-dependent AFL ( $8 \%$ vs. $0 \% \mathrm{P}=0.041$ ). There was no significant difference in the frequency of focal AT between groups (Table 13.) 


\begin{tabular}{|llllll|}
\hline Atrial tachycardia & \multicolumn{2}{l|}{ Atrial incision } & & \\
\cline { 2 - 6 } & $\begin{array}{l}\text { RA } \\
\text { cannulation } \\
(\mathbf{n = 2 0})\end{array}$ & $\begin{array}{l}\text { RA atriotomy } \\
(\mathbf{n = 3 2})\end{array}$ & $\begin{array}{l}\text { Transseptal LA atriotomy } \\
(\mathbf{n = 4 1 )}\end{array}$ & Direct LA atriotomy & P value \\
& $95 \%$ & $94 \%$ & $83 \%$ & $71 \%$ & 0.191 \\
\hline CTI-dependent AFL & $15 \%$ & $41 \%$ & $73 \%$ & $57 \%$ & $<0.001$ \\
Non-CTI-dependent AFL & $15 \%$ & $34 \%$ & $49 \%$ & $0 \%$ & 0.013 \\
RA incisional & $0 \%$ & $3 \%$ & $17 \%$ & $57 \%$ & 0.001 \\
Perimitral & $0 \%$ & $0 \%$ & $7 \%$ & $14 \%$ & 0.147 \\
LA roof-dependent & $0 \%$ & $13 \%$ & $15 \%$ & $14 \%$ & 0.365 \\
Focal AT & & & & \\
\hline
\end{tabular}

Table 13. Comparison of AT mechanisms between surgical groups, RA right atrium, LA left atrium, CTI cavotricuspid isthmus, AFL atrial flutter, AT atrial tachycardia. 


\section{Multivariate analysis}

Age, sex, history of hypertension, congenital heart disease, ischemic heart disease, valve surgery and preprocedural echocardiographic dimensions (LA diameter, end-diastolic, and end-systolic left ventricular diameter) were analyzed in respect to the different AT mechanisms. When any of these parameters was significantly related to a specific AT, it was included in multivariate analysis using logistic regression. No predictor was recognized for CTI-dependent AFL, which was equally prevalent in all groups. With non-CTI-dependent AFL, surgical group and larger LA diameter showed significant correlation, but only surgical group proved to be an independent predictor in multivariate analysis $(\mathrm{P}<0.001)$. Only surgical group was associated with RA incisional tachycardia $(\mathrm{P}=0.011)$. Perimitral AFL besides being related to surgical group showed a positive association with LA diameter $(\mathrm{P}=0.039)$ and valve surgery $(\mathrm{P}=0.016)$, negative association with left ventricular enddiastolic and end-systolic diameters $(\mathrm{P}=0.030$ and $\mathrm{P}=0.043$, respectively). With multivariate analysis surgical group and end-systolic diameter remained independent predictors of perimitral AFL $(\mathrm{P}=0.019$ and $\mathrm{P}=0.036$, respectively). Left atrial atriotomy was the only predictor for LA roof-dependent AFL $(\mathrm{P}=0.041)$. No predictor was identified for focal AT.

\section{$\underline{\text { Radiofrequency ablation }}$}

In the case of CTI-dependent AFLs, linear ablation of the CTI was performed until termination of the tachycardia and establishment of bidirectional conduction block of the CTI. This was successful in 87 of $88(99 \%)$ cases, while in one case AFL terminated, but CTI block could not be achieved. All 34 RA incisional tachycardias were ablated successfully: tachycardia terminated in 28 cases when a linear ablation lesion was placed between the atriotomy and the inferior vena cava, in one case between the atriotomy and a septal scar, in two cases between the atriotomy and the tricuspid ring, and in three cases when narrow channels were closed within the scar of the lateral RA atriotomy. Complete conduction block along these lines was confirmed by electroanatomical mapping demonstrating an activation detour during pacing close to the line in all cases. In case of perimitral flutter linear ablation at the mitral isthmus was attempted first, except for one case where a narrow channel was noted between the anteroseptal atriotomy scar and the mitral annulus. A single radiofrequency lesion at this site terminated the tachycardia and rendered it noninducible (Fig.3.). Mitral 
isthmus ablation led to the termination of the arrhythmia and development of conduction block in six of the other $11(54.5 \%)$ cases. In two of the remaining five cases epicardial ablation in the CS at the level of the endocardial ablation line led to conduction block, while in three cases this was also unsuccessful. In one of these cases, the tachycardia terminated, but conduction block could not be achieved, and in one other case, an anterior ablation line from the mitral annulus to the left upper pulmonary vein terminated the AFL. Perimitral AFL of one patient could not be terminated by ablation. Three off our LA roof-dependent AFLs were ablated by linear ablation between upper pulmonary veins and one by mitral isthmus ablation. All focal ATs were successfully terminated by ablation and rendered noninducible.

\section{Follow up}

Twenty-one patients were lost to follow up; the rest were followed for $19 \pm 15$ months. Seventeen patients (22\%) had recurrent AFL, and 21 (27\%) had atrial fibrillation during follow up. Recurrent AFL was more common in patients who had operations involving the LA ( $8 \%$ vs. $30 \%, P=0.015)$. Patients who developed atrial fibrillation during follow up had larger LA diameter (57 vs. $52 \mathrm{~mm}, \mathrm{P}=0.028$ ). No other parameters showed an association with recurrent AFL or atrial fibrillation. 


\section{Discussion}

1.

We investigated clinical and echocardiographic parameters for AF recurrence after RFA in patients with persistent and longstanding persistent nonvalvular AF, and found LA size measurements in the 4C view, including length, area, and both maximum and minimum volumes, to be superior to any other diastolic function parameters and directly measured LA pressure in association with recurrence in this patient population.

Echocardiography is a very important tool to evaluate AF patients. Several studies showed that LA enlargement was a well-recognized risk factor for recurrence after cardioversion and catheter ablation. ${ }^{33-35}$ Recent studies showed that noninvasive assessment of LV filling pressure can help to predict recurrence after SR is restored. ${ }^{12}$

The restoration of SR is much more challenging in patients with persistent and longstanding AF, probably due to more advanced remodeling. The previous studies evaluated the predictors in a mixed study population including paroxysmal, persistent, and longstanding persistent AF patients. ${ }^{36,37,12,13}$ Our study focused on patients with persistent and longstanding persistent AF and we evaluated clinical, invasive, and noninvasive diastolic function parameters. Our finding highlighted the significance of LA enlargement in ablation outcome in these patients.

\section{Clinical Parameters and Recurrence of AF}

We found that the duration of AF prior RFA was the only significant clinical parameter correlated with AF recurrence. A recent study also showed that the duration of AF is an important predictor of SR maintenance after RFA, although the literature is controversial regarding this finding. ${ }^{11,36,38}$ Longstanding AF may lead to atrial fibrosis and the development of AF substrate through structural remodeling. AF associated structural and pathophysiological changes are very important factors that promote remodeling ${ }^{10}$ and might have a role in catheter ablation outcome.

\section{Echocardiographic Parameters and Recurrence of AF}

Our study is the first to evaluate both the invasive and noninvasive diastolic function parameters in association with AF recurrence after RFA in one setting in patients with persistent AF. Recent studies showed that diastolic function parameters might be able to predict AF recurrences after PVI; however, these parameters were assessed individually. LAV 
assessed by computer tomography was shown to be a potential predictor of AF recurrence. ${ }^{14,36}$ The ratio of E/E'has been shown to be a useful parameter to predict SR maintenance. ${ }^{12} \mathrm{LA}$ remodeling plays an important role in initiation and perpetuation of AF. The reversibility of this remodeling depends on the strength and the duration of exposure to stressors. The most common stressors of atrial myocytes are volume/pressure overload and tachycardia. Increased volume/pressure overload leads to chamber dilatation and stretch of the myocardium,

providing a substrate for AF. ${ }^{10}$ Supporting this concept, LA size has been shown to correspond with atrial fibrosis ${ }^{39}$ and the development of fibrosis leads to LA dilation and subsequently AF. ${ }^{40}$ Although the mean LAP was not significantly different in patients with and without arrhythmia recurrence, the structural changes shown by LAV parameters may reflect duration of exposure. We suspect that the $4 \mathrm{C}$ view measurements are superior for LA size because the entire LA is well visualized in this view due to optimized ultrasound probe position for this view as compared to the $2 \mathrm{C}$ or the parasternal long axis view. A recent study showed minimal LAV might be superior to maximum LAV for prediction of first AF and/or atrial flutter in an elderly cohort. ${ }^{41}$ Our results substantiate this study in a younger population with persistent AF. LA minimum volume might be less dependent on loading conditions than LA maximum volume, and is therefore a better presentation of LA size.

\section{$\underline{2}$.}

The major finding of this study is that the time to AF recurrence after catheter ablation dramatically influences clinical outcome. The longer the time to AF recurrence, the more likely that a patient will have a good clinical course with infrequent additional AF episodes, more drug responsiveness and an excellent response to repeat catheter ablation.

\section{Comparison with previous studies}

The benign pattern of very late AF recurrences has been previously suggested. ${ }^{42,43}$ However, these prior studies were limited by small size and included few patients with nonparoxysmal $\mathrm{AF}^{42,43}$ Our study is unique in reporting long-term outcome of a large cohort of patients with paroxysmal and nonparoxysmal AF with after ablation AF recurrences after the initial procedure. Our study is also unique in its attempt to define outcome based on the time of recurrence after ablation by dividing the population into 3 clinically meaningful subgroups: early recurrences (3-6 months), late recurrences (6-12 months), and very late recurrences (>12 months) after ablation. Furthermore, we sought to identify (1) patients with only rare AF 
episodes during the long-term follow-up after the first AF recurrence and (2) responsiveness to AADs and repeat catheter ablation, as indices of a more "benign" clinical course. Patients with recurrences in the first 3-6 months after ablation represented the largest group in our study. These patients had the least favorable outcomes in that most had subsequent frequent $\mathrm{AF}$ and did not respond to new AADs when tried. Most of these patients were already on AADs (59\%), and a decision to pursue repeat ablation was frequently chosen as the preferred therapeutic option. The outcome was worse in comparison to the other groups after the second ablation attempt, and significantly more ablation procedures were necessary in this group to achieve a level of AF control comparable to those with late rrecurrences. Patients with recurrences between 6 and 12 months have been previously designated as both early recurrences and late recurrences. ${ }^{44,45}$ However, we showed that this group of patients is clinically different from patients with recurrence between 3 and 6 months after ablation and those with recurrence $>12$ months after ablation. This group represents an "intermediate" group, with outcomes after recurrence falling between those of patients who had $\mathrm{E}$ and VL AF recurrences. In this large consecutive group of patients, we demonstrate that patients with VL recurrence are less common than those who recur earlier after ablation. Our incidence of $6.4 \%$ for late AF is comparable to the $5.0 \%-7.8 \%$ reported in smaller series. ${ }^{46}$ Importantly, additional AF recurrences after 1 year tend to occur as rare episodes and have a better response to AADs. Even patients with recurrence after a year who had frequent AF episodes responded better to AADs than their counterpart patients whose AF recurred earlier after ablation. In addition, patients with very late recurrences underwent less repeat ablations, and when performed, the results of repeat ablation were associated with better clinical outcome. Most patients ultimately could be maintained off AADs. It is important to note that a significant number of patients with recurrence after AF ablation had a good clinical outcome after a single procedure, although they were not completely arrhythmia-free. In patients with AF recurrences after 6 and 12 months who did not undergo repeat ablation, our data show $77 \%$ and $95 \%$ of these patients, respectively, experience no or very sporadic AF episodes after their initial recurrence, with long arrhythmia-free intervals (Figure 9).

\section{Other factors associated with the time of recurrence after AFablation and clinical outcome}

There was a higher incidence of recurrences in the blanking period in patients with $\mathrm{E}$ recurrences in comparison to those with $\mathrm{L}$ or $\mathrm{VL}$ recurrences $(82 \%$ vs. $54 \% ; \mathrm{P}=0.001)$. This suggests that patients included in the E group represent a subset of patients who may have already developed acute PV reconnection. Of interest, patients with E recurrences also had a 
longer history of AF in comparison to those with VL recurrences, which may imply that these patients have a more arrhythmogenic substrate. It is well described that AF promotes atrial remodeling, which leads to a more resistant substrate for treatment. ${ }^{10}$ Longer duration of AF has been shown to be an independent predictor of recurrences after ablation in previous studies, ${ }^{45}$ and we have shown that it is also an independent predictor of frequent arrhythmia episodes after the initial recurrence and repeat ablation. In addition, recurrence during the blanking period predicted more frequent subsequent $\mathrm{AF}$ episodes and a more troublesome clinical course. Nonparoxysmal AF is associated with worse outcome after catheter ablation. However, it is of interest that both patients with paroxysmal and nonparoxysmal AF in the $\mathrm{E}$ recurrence group had a poorer response to AADs, were unlikely to have only rare AF during subsequent follow-up (Figure 1), and had a worse outcome after repeat ablation. These findings highlight the primary influence of the time of recurrence on clinical outcome regardless of AF type.

\section{Mechanistic implications for the influence of the time of recurrence after ablation on subsequent clinical outcome}

While $97 \%$ of the patients undergoing repeat AF ablation have reconnected PVs, it could be that patients with late recurrence have less extensive areas of reconnection, which result in more limited PV to LA conduction and, consequently, fewer recurrences and better response to AADs. Verma et $\mathrm{al}^{47}$ reported that patients who maintained sinus rhythm on AADs had a significant conduction delay between reconnected PVs and the LA whereas patients who did not respond to AADs had only a minimal conduction delay. In the present study, we did not find differences related to primary ablation strategy and time of recurrence, possibly because of the uniformity of the ablation procedure during the study period.

\section{$\underline{3 .}$}

This study has shown that the finding of non-CTI-dependent AFL at electrophysiology study performed for clinical AT occurring late after open heart surgery is strongly associated with the atrial incisions applied during the operation. While CTI-dependent AFL is the most common AT in all groups, a non-CTI-dependent, atypical AFL becomes progressively more common with more extensive atrial incisions. Of the different non-CTI-dependent circuits, RA incisional tachycardias dominant after surgery involving the RA, while a perimitral or LA roof-dependent circuit can be expected after LA operations. Focal AT was not correlated with 
atriotomy, but - other than the typical predilection sites - showed a tendency to originate from the vicinity of surgical scar.

\section{Comparison with previous studies}

Lukac et al. ${ }^{48}$ could not show a difference in the distribution of different atrial tachycardias developing after cardiac surgery for a wide range of indications. They included patients with complex congenital heart disease, heart transplantation, and maze procedure and adopted a classification for AT based only on the mechanism ("incisional" and "not incisional") rather than the location of the circuit. Our patients represent a more routinely encountered population with postoperative arrhythmias presenting to a center not specifically involved in the management of patients after heart transplantation. Furthermore, our classification of AT is different: not only by mechanism (focal or AFL) but by the common location (e.g., RA free wall) and ablation strategy of the AT. For the above reasons, our results are not directly comparable to those of Lukac et al. Nevertheless, they also show the most frequent AT late after cardiac surgery to be CTI-dependent AFL. Also, the reported frequencies of RA incisional tachycardia among their patients comparable to our groups (after right lateral atriotomy and transseptal approach to the mitral valve) are very similar to our results (33\% and $40 \%$, respectively). ${ }^{48}$ In the largest reported series of postoperative AFL, Aktas et al. found CTI-dependent AFL to be most common. Similar to our results, the indication for surgery (valve- or onvalve-related) did not influence the type of AFL. However, the association between atrial incisions and type of AFL was not specifically studied. ${ }^{23}$

\section{Cavotricuspid isthmus-dependent postoperative flutter}

Functional and/or fixed block to impulse propagation between the venae cavae favors the development of this arrhythmia after heart surgery. ${ }^{49,50}$ Many studies have found the dominant role of the CTI in postoperative AFL mechanism ${ }^{23,24,48}$, while others have shown incisional tachycardia, depending on an isthmus bordered by an atrial incision to be most frequent. ${ }^{19}, 25$, ${ }^{51-53}$ In the study by Anné et al. ${ }^{18}$, CTI-dependent flutter predominated in the congenital heart disease group, while RA incisional tachycardia was more frequent in a group of patients with acquired heart disease and mostly mitral valve operations. According to our results, CTIdependent AFL is the most common AT after open heart surgery in all groups, and the relative frequencies of CTI-dependent and non-CTI dependent AFL circuits are related not to the underlying heart disease but more to the atrial incisions created during the operation. 


\section{Right atrial incisional tachycardia}

While an intercaval line of block favors classical AFL development, a sufficiently long, fixed barrier in the RA free wall may predispose to incisional tachycardia. ${ }^{49,54} \mathrm{~A}$ surgical incision on the RA free wall facilitates the development of this arrhythmia, however, the extent and position of the lesion seems to be an important factor. In this study, among patients who underwent venous cannulation only (at the RA appendage), 15\% had RA incisional tachycardia, but among patients who had a long RA free wall atriotomy, this AT was seen in $43 \%$ (Fig. 2.). This latter group comprised patients after open cannulation of the coronary sinus for retrograde cardioplegia, a procedure reported previously to be arrhythmogenic ${ }^{55}$ and to favor RA incisional tachycardia. ${ }^{25}$ Also included in this group were patients after atrial or ventricular septal defect closure or tricuspid valve operation. These operations are also known for their potential to result in late RA incisional AFL. ${ }^{56}$ This tachycardia was found to be most common (49\% of patients) when the RA free wall atriotomy was prolonged to the roof of the RA, between the superior caval vein and the tricuspid annulus, and further onto the interatrial septum during transseptal LA atriotomy. The longer incision may favor peri-lesional AFL by a longer path length of reentry, more likely development of slow conduction at sites of reconnection along the long lesion or in the longer corridor between the incision and the tricuspid annulus. ${ }^{57}$ Also, the more anteriorly placed incision is more likely to cause periincisional reentry as shown by Tomita et al. ${ }^{49}$ The rotating wavefront either uses the isthmus between the incision and the inferior caval vein, or a channel of reconnection along the lesion, but always uses at least some part of the corridor between the incision and the tricuspid annulus. Reconnection can occur anywhere along this line thereby connecting the two sides of the incision and closing the reentrant circuit, which can be located both septally ${ }^{58}$ or more on the free wall ${ }^{57}$ in this group of patients. Isolated channels or a narrow corridor might allow for focal ablation. ${ }^{19,} 58$ Otherwise, the arrhythmia can be terminated by a linear lesion connecting the incision to another barrier: the inferior vena cava or tricuspid annulus. ${ }^{60}$ This suggests that extension of the incision towards the inferior caval vein and/or surgical ablation to connect with the tricuspid annulus at the time of surgery might prove to be effective against the future occurrence of this arrhythmia.

\section{Left atrial flutters}

The LA is a much less common site than the RA for AFL, both in patients with and without prior cardiac surgery. ${ }^{23}$ Nevertheless, the finding of LA flutter is more frequent after surgery. ${ }^{23}$ Similarly to classical peritricuspid flutter after RA incisions, surgical scar in the LA 
may pave the way for perimitral reentry. This is supported by the fact that this arrhythmia was seen almost exclusively after operations involving the LA in this series. However, similar to previous experience ${ }^{61,62}$, roof-dependent LA flutters also occurred in this group of patients, using the pulmonary veins and LA scar as the central obstacle.

\section{Focal atrial tachycardia}

Focal AT was an uncommon finding in these patients late after open heart surgery, similarly to previously reported series of operated congenital heart disease. ${ }^{63}$ The arrhythmia originated from the vicinity of surgical scar in more than one third of the cases - a previously reported finding after operation for congenital heart disease. ${ }^{22}$ Other sites of origin of focal AT, including the AV rings and pulmonary veins, are typical predilection sites in normal hearts also. $^{64}$ 


\section{Conclusion}

Atrial fibrillation and atrial flutter play an essential role in atrial remodeling, and atrial tachycardias lead to several adverse clinical consequences such as impairment of quality of life, embolic events and congestive heart failure. Catheter ablation is a very important tool in the treatment of these atrial tachycardias, hereby it helps to slow down and inhibit the process of remodeling. Optimal patient selection, the detailed knowledge of atrial anatomy and the type atrial incisions applied during open heart surgery could be the key to the success of the procedure. 


\section{New observations}

1. Results of this study demonstrate the importance of indexed LAV in association with recurrence in patients with persistent and longstanding persistent AF undergoing RFA. The duration of persistent AF also has a negative impact on AF ablation success. These findings, along with the results from prior studies, suggest that PVI success remains difficult to predict, but the longer duration of AF and larger indexed LAV are strongly associated with recurrence and should be incorporated into the recurrence stratificationin patients with persistent and longstanding persistent AF.

2. In patients with AF recurring after catheter ablation, the time to recurrence is a significant predictor of subsequent clinical outcome. Patients with later recurrences (1) are more likely to have sporadic AF episodes, (2) can be better managed with AADs, and (3) have better results after repeat ablation.

3. For the electrophysiologist treating operated patients with AT, it is very important to review surgical records and clarify the specific atriotomy used, since different approaches result in different AT mechanisms. Modification of the surgical technique might be effective against the future occurrence of AT in these patients. For example, avoiding RA incision by using direct LA atriotomy instead of the transseptal approach has been shown to be associated with less frequent occurrence of AT. ${ }^{65}$ 


\section{References}

1. Olsson LG, Swedberg K, Ducharme A, Granger CB, Michelson EL, McMurray JJ, Puu M, et al. Atrial fibrillation and risk of clinical events in chronic heart failure with and without left ventricular systolic dysfunction: Results from the Candesartan in Heart failure-Assessment of Reduction in Mortality and morbidity (CHARM) program. J Am Coll Cardiol 2006; 47:1997-2004.

2. M Allessie, J Ausma, U Schotten, Electrical, contractile and structural remodeling during atrial fibrillation, Cardiovascular Research 54 (2002) 230-246, Review

3. Oral H, Pappone C, Chugh A, Good E, Bogun F, Pelosi F Jr., Bates ER, et al. Circumferential pulmonary-vein ablation for chronic atrial fibrillation. N Engl J Med 2006; 354:934-941.

4. Hässaguerre M, Jäis P, Shah DC, Takahashi A, Hocini M, Quiniou G, Garrigue S, et al. Spontaneous initiation of atrial fibrillation by ectopic beats originating in the pulmonary veins. N Engl J Med 1998; 339:659-666.

5. Riccardo Cappato, Hugh Calkins et al, Updated Worldwide Survey on the Methods, Efficacy, and Safety of Catheter Ablation for Human Atrial Fibrillation, Circ Arrhythm Electrophysiol. 2010;3:32-38.

6. Weerasooya R, Khairy P, Litaien J, et al. Catheter ablation for atrial fibrillation. Are results maintained at 5 years of follow up? J Am Coll Cardiol 2011;57: 160-166.

7. Tzou WS, Marchlinski FE, Zado ES, et al. Long-term outcome after successful catheter ablation of atrial fibrillation. Circ Arrhythm Electrophysiol 2010;3: 237-242.

8. To AC, Flamm SD, Marwick TH, Klein AL. Clinical utility of multimodality LA imaging: assessment of size, function, and structure. JACC Cardiovasc Imaging. 2011 Jul;4 (7):788-98.

9. Kim SJ, Choisy SC, Barman P, Zhang H, Hancox JC, Jones SA, James AF. Atrial remodeling and the substrate for atrial fibrillation in rat hearts with elevated afterload. Circ Arrhythm Electrophysiol. 2011 Oct; 4 (5):761-9

10. Casaclang-Verzosa G, Gersh BJ, Tsang TS. Structural and functional remodeling of the left atrium: clinical and therapeutic implications for atrial fibrillation. J Am Coll Cardiol.2008 Jan 1; 51 (1):1-11. 
11. den Uijl DW, Delgado V, Tops LF, Ng AC, Boersma E, Trines SA, Zeppenfeld K, et al. Natriuretic peptide levels predict recurrence of atrial fibrillation after radiofrequency catheter ablation. Am Heart J 2011; 161:197-203.

12. Li C, Ding X, Zhang J, Zhou C, Chen Y, Rao L. Does the E/e' index predict the maintenance of sinus rhythm after catheter ablation of atrial fibrillation? Echocardiography 2010; 27:630-636.

13. Shin SH, Park MY, Oh WJ, Hong SJ, Pak HN, Song WH, Lim DS, et al. Left atrial volume is a predictor of atrial fibrillation recurrence after catheter ablation. J Am Soc Echocardiogr 2008; 21:697-702.

14. von Bary C, Dornia C, Eissnert C, Nedios S, Roser M, Hamer OW, Gerds-Li JH, et al. Predictive value of left atrial volumemeasured by non-invasive cardiac imaging in the treatment of paroxysmal atrial fibrillation. J Interv Card Electrophysiol 2012; 34:181188.

15. Caputo M, Urselli R, Capati E, Navarri R, Sinesi L, Furiozzi F, Ballo P, et al. Usefulness of left ventricular diastolic dysfunction assessed by pulsed tissue Doppler imaging as a predictor of atrial fibrillation recurrence after successful electrical cardioversion. Am J Cardiol 2011; 108:698-704.

16. M. R. Franz, P. L. Karasik, C. Li, J. Moubarak, M. Chavez, Electrical Remodeling of the Human Atrium: Similar Effects in Patients With Chronic Atrial Fibrillation and Atrial Flutter JACC 1997:1785-92

17. Hammon, J. W. Extracorporeal circulation: Perfusion system. In L. H. Cohn (Ed.), Cardiac surgery in the adult (2008, pp. 350-370). New York: McGraw-Hill.

18. Anné, W. van Rensburg, H., Adams, J., Ector, H., Van de Werf, F., \& Heidbüchel, H. Ablation of post-surgical intra-atrial reentrant tachycardia. Predilection target sites and mapping approach. European Heart Journal, 2002, 23 (20), 1609-1616.

19. Nakagawa, H., Shah, N., Matsudaira, K., Overholt, E., Chandrasekaran, K., Beckman, K. J., Spector, P., et al. Characterization of reentrant circuit in macroreentrant right atrial tachycardia after surgical repair of congenital heart disease: Isolated channels $\mathbf{J}$ Interv Card Electrophysiol between scars allow "focal" ablation. Circulation, 2001, 103, 699-709.

20. Love, B. A., Collins, K. K.,Walsh, E. P., Triedman, J. K. Electroanatomic characterization of conduction barriers in sinus/atrially paced rhythm and association with intra-atrial reentrant tachycardia circuits following congenital heart disease surgery. Journal of Cardiovascular Electrophysiology, 2001, 12(1), 17-25. 
21. Shah, D., Jaïs, P., Haïssaguerre, M. Electrophysiological evaluation and ablation of atypical right atrial flutter. Cardiac Electrophysiology Review, 2002,6(4), 365-370.

22. de Groot, N. M., Zeppenfeld, K., Wijffels, M. C., Chan, W. K., Blom, N. A., van der Wall, E. E., Schalij, M. J. Ablation of focal atrial arrhythmia in patients with congenital heart defects after surgery: Role of circumscribed areas with heterogeneous conduction. Heart Rhythm, 2006, 3, 526-535.

23. Aktas, M. K., Khan, M. N., Di Biase, L., Elayi, C., Martin, D., Saliba, W., Cummings, J., Schweikert, R., Natale, A. Higher rate of recurrent atrial flutter and atrial fibrillation following atrial flutter ablation after cardiac surgery. Journal of cardiovascular Electrophysiology, 2010, 21(7), 760-765.

24. Chan, D. P., Van Hare, G. F., Mackall, J. A., Carlson, M. D., Waldo, A. L. Importance of atrial flutter isthmus in postoperative intra-atrial reentrant tachycardia. Circulation, 2000, 102(11), 1283-1289.

25. Verma, A., Marrouche, N. F., Seshadri, N., Schweikert, R. A., Bhargava, M., Burkhardt, J. D., Kilicaslan, F., et al. Importance of ablating all potential right atrial flutter circuits in postcardiac surgery patients. Journal of the American College of Cardiology, 2004, 44 (2), 409-414.

26. Calkins H, Kuck KH, Cappato R, Brugada J, Camm AJ, Chen SA, Crijns HJ, et al. 2012 HRS/EHRA/ECAS expert consensus statement on catheter and surgical ablation of atrial fibrillation: Recommendations for patient selection, procedural techniques, patient management and follow-up, definitions, endpoints, and research trial design: a report of the Heart Rhythm Society (HRS) Task Force on Catheter and Surgical Ablation of Atrial Fibrillation. Developed in partnership with the European Heart Rhythm Association (EHRA), a registered branch of the European Society of Cardiology (ESC) and the European Cardiac Arrhythmia Society (ECAS); and in collaboration with the American College of Cardiology (ACC), American Heart Association (AHA), the Asia Pacific Heart Rhythm Society (APHRS), and the Society of Thoracic Surgeons (STS). Endorsed by the governing bodies of the American College of Cardiology Foundation, the American Heart Association, the European Cardiac Arrhythmia Society, the European Heart Rhythm Association, the Society of Thoracic Surgeons, the Asia Pacific Heart Rhythm Society, and the Heart Rhythm Society. Heart Rhythm 2012; 9:632-696, e621. 
27. Leong-Sit P, Zado E, Callans DJ, Garcia F, Lin D, Dixit S, Bala R, et al. Efficacy and risk of atrial fibrillation ablation before 45 years of age. Circ Arrhythm Electrophysiol $2010 ; 3: 452-457$.

28. Dixit S, Marchlinski FE, Lin D, Callans DJ, Bala R, Riley MP, Garcia FC, et al. Randomized ablation strategies for the treatment of persistent atrial fibrillation: RASTA study. Circ Arrhythm Electrophysiol 2012; 5:287-294.

29. Gerstenfeld EP, Dixit S, Callans D, Rho R, Rajawat Y, Zado E, Marchlinski FE. Utility of exit block for identifying electrical isolation of the pulmonary veins. $\mathbf{J}$ Cardiovasc Electrophysiol 2002; 13:971-979.

30. Gerstenfeld EP, Sauer W, Callans DJ, et al. Predictors of success after selective pulmonary vein isolation of arrhythmogenic pulmonary veins for treatment of atrial fibrillation. Heart Rhythm 2006;3:165-170.

31. Roux J-F, Zado E, Callans J, et al. Antiarrhythmics after ablation of atrial fibrillation (5A Study). Circulation 2009;120:1036-1040.

32. Lang RM, Bierig M, Devereux RB, Flachskampf FA, Foster E, Pellikka PA, Picard $\mathrm{MH}$, et al. Recommendations for chamber quantification: A report from the American Society of Echocardio-graphy's Guidelines and Standards Committee and the Chamber Quantification Writing Group, developed in conjunction with the European Association of Echocardiography, a branch of the European Society of Cardiology. J Am Soc Echocardiogr 2005; 18:1440-1463.

33. Marchese P, Malavasi V, Rossi L, Nikolskaya N, Donne GD, Becirovic M, Colantoni A, et al. Indexed left atrial volume is superior to left atrial diameter in predicting nonvalvular atrial fibrillation recurrence after successful cardioversion: A prospective study. Echocardiography 2012; 29:276-284.

34. Marchese P, Bursi F, Delle Donne G, Malavasi V, Casali E, Barbieri A, Melandri F, et al. Indexed left atrial volume predicts the recurrence of non-valvular atrial fibrillation after successful cardioversion. Eur J Echocardiogr 2011; 12:214-221.

35. Abecasis J, Dourado R, Ferreira A, Saraiva C, Cavaco D, Santos KR, Morgado FB, et al. Left atrial volume calculated by multidetector computed tomography may predict successful pulmonary vein isolation in catheter ablation of atrial fibrillation. Europace 2009; 11:1289-1294.

36. Hof I, Chilukuri K, Arbab-Zadeh A, Scherr D, Dalal D, Nazarian S, Henrikson C, et al. Does left atrial volume and pulmonary venous anatomy predict the outcome of 
catheter ablation of atrial fibrillation? J Cardiovasc Electrophysiol 2009; 20:10051010.

37. Lee SH, Tai CT, Hsieh MH, Tsai CF, Lin YK, Tsao HM, Yu WC, et al. Predictors of early and late recurrence of atrial fibrillation after catheter ablation of paroxysmal atrial fibrillation. J Interv Card Electrophysiol 2004; 10:221-226.

38. Lin D, Frankel DS, Zado ES, Gerstenfeld E, Dixit S, Callans DJ, Riley M, et al. Pulmonary vein antral isolation and nonpulmonary vein trigger ablation without additional substrate modification for treating longstanding persistent atrial fibrillation. J Cardiovasc Electrophysiol 2012; 23:806-813.

39. Kataoka T, Hamasaki S, Inoue K, Yuasa T, Tomita K, Ishida S, Ogawa M, et al. Left atrium volume index and pathological features of left atrial appendage as a predictor of failure in postoperative sinus conversion. J Cardiol 2010; 55:274-282.

40. Burstein B, Nattel S. Atrial fibrosis: Mechanisms and clinical relevance in atrial fibrillation. J Am Coll Cardiol 2008; 51:802-809.

41. Fatema K, Barnes ME, Bailey KR, Abhayaratna WP, Cha S, Seward JB, Tsang TS. Minimum vs. maximum left atrial volume for prediction of first atrial fibrillation or flutter in an elderly cohort: A prospective study. Eur J Echocardiogr 2009; 10:282286.

42. Hsieh MH, Tai CT, Tsai CF, et al. Clinical outcome of very late recurrence of atrial fibrillation after catheter ablation of paroxysmal atrial fibrillation. J Cardiovasc Electrophysiol 2003;14:598-601.

43. Shah AN, Mittal S, Sichrovsky TC, et al. Long-term outcome following successful pulmonary vein isolation: pattern and prediction of very late recurrence. J. Cardiovasc Electrophysiol 2008;19:661-667.

44. Hsieh MH, Tai CT, Lee SH, et al. The different mechanisms between late and very late recurrences of atrial fibrillation in patients undergoing a repeated catheter ablation. J Cardiovasc Electrophysiol 2006;17:231-235.

45. Themistoclakis S, Schweikert R, Saliba W, et al. Clinical predictors and relationship between early and late atrial tachyarrhythmias after pulmonary vein antrum isolation. Heart Rhythm 2008;5:679-685.

46. Mainigi SK, Sauer WH, Cooper JM, et al. Incidence and predictors of very late recurrence of atrial fibrillation after ablation. J. Cardiovasc Electrophysiol 2007;18:69-74. 
47. Verma A, Kilicaslan F, Pissano E, et al. Response of atrial fibrillation to pulmonary vein antrum isolation is directly related to resumption and delay of pulmonary vein conduction. Circulation 2005;112:627-635.

48. Lukac, P., Pedersen, A. K., Mortensen, P. T., Jensen, H. K., Hjortdal, V., Hansen, P. S. Ablation of atrial tachycardiaafter surgery for congenital and acquired heart disease using an electroanatomic mapping system: Which circuits to expect in which substrate? Heart Rhythm, 2005, 2(1), 64-72.

49. Tomita, Y., Matsuo, K., Sahadevan, J., Khrestian, C. M., Waldo, A. L. Role of functional block extension in lesion-related atrial flutter. Circulation, 2001, 103(7), $1025-1030$.

50. Bui, H. M., Khrestian, C. M., Ryu, K., Sahadevan, J., Waldo, A. L. Fixed intercaval block in the setting of atrial fibrillationpromotes the development of atrial flutter. Heart Rhythm, 2008, 5(12),1745-1752.

51. Akar, J. G., Kok, L. C., Haines, D. E., DiMarco, J. P., Mounsey, J. P. Coexistence of type I atrial flutter and intra-atrial reentrant tachycardia in patients with surgically corrected congenital heart disease. Journal of the American College of Cardiology, 2001, 38(2), 377-384.

52. Hebe, J., Hansen, P., Ouyang, F., Volkmer, M., Kuck, K. H. Radiofrequency catheter ablation of tachycardia in patients with congenital heart disease. Pediatric Cardiology, 2000, 21(6), 557-575.

53. Markowitz, S. M., Brodman, R. F., Stein, K. M., Mittal, S., Slotwiner, D. J., Iwai, S., Das, M. K., et al. Lesional tachycardias related to mitral valve surgery. Journal of the American College of Cardiology, 2002, 39(12), 1973-1983.

54. Waldo, A. L. Mechanisms of atrial flutter and atrial fibrillation: Distinct entities or two sides of a coin? Cardiovascular Research, 2002, 54(2), 217-229.

55. Mori, S. S., Fujii, G. G., Ishida, H. H., Tomari, S. S., Matsuura, A. A., Yoshida, K. K. Atrial flutter after coronary arterybypass grafting: Proposed mechanism as illuminated by independent predictors. Annals of Thoracic and Cardiovascular Surgery, 2003, 9, $50-56$.

56. Magnin-Poull, I., De Chillou, C., Miljoen, H., Andronache, M., Aliot, E. Mechanisms of right atrial tachycardia occurring late after surgical closure of atrial septal defects. Journal of Cardiovascular Electrophysiology, 2005, 16(7), 681-687. 
57. Lukac, P., Hjortdal, V., Pedersen, A. K., Jensen, H. K., Mortensen, P. T., Hansen, P. S. The superior transseptal surgicalapproach to mitral valve creates slow conduction. Pacing and Clinical Electrophysiology, 2006, 29(7), 719-726.

58. Roberts-Thomson, K. C., Kalman, J. M. Right septal macroreentrant tachycardia late after mitral valve repair: Importance of surgical access approach. Heart Rhythm, 2007, $4(1), 32-36$.

59. Pap, R., Makai, A., Sághy, L. Post-incisional right atrial tachycardia eliminated by a single radiofrequency lesion. Journal of Interventional Cardiac Electrophysiology, 2007, 19(2), 73-76.

60. Snowdon, R. L., Balasubramaniam, R., Teh, A. W., Haqqani, H. M., Medi, C., Rosso, R., Vohra, J. K., Kistler, P. M., Morton, J. B.,Sparks, P. B., Kalman, J. M. Linear ablation of right atrial free wall flutter: Demonstration of bidirectional conduction block as an endpoint associated with long-term success. Journal of Cardiovascular Electrophysiology, 2010, 21(5), 526-531.

61. Jaïs, P., Shah, D. C., Haïssaguerre, M., Hocini, M., Peng, J. T., Takahashi, A., Garrigue, S., et al. Mapping and ablation of left atrial flutters. Circulation, 2000, 101(25), 2928-2934.

62. Ouyang, F., Ernst, S., Vogtmann, T., Goya, M., Volkmer, M., Schaumann, A., Bänsch, D., et al. Characterization of reentrant circuits in left atrial macroreentrant tachycardia: Critical isthmus block can prevent atrial tachycardia recurrence. irculation, 2002, 105(16), 1934-1942.

63. de Groot, N. M., Atary, J. Z., Blom, N. A., Schalij, M. J. Long-term outcome after ablative therapy of postoperative atrialtachyarrhythmia in patients with congenital heart disease and characteristics of atrial tachyarrhythmia recurrences. Circulation. Arrhythmia and Electrophysiology, 2010, 3(2), 148-154.

64. Chen, S. A., Tai, C. T., Chiang, C. E., Ding, Y. A., Chang, M. S. Focal atrial tachycardia: Reanalysis of the clinical andelectrophysiologic characteristics and prediction of successful radiofrequency ablation. Journal of Cardiovascular Electrophysiology, 1998, 9(4), 355-365.

65. Lukac, P., Hjortdal, V. E., Pedersen, A. K., Mortensen, P. T., Jensen, H. K., Hansen, P. S. Atrial incision affects the incidence of atrial tachycardia after mitral valve surgery. The Annals of Thoracic Surgery, 2006, 81(2), 509-513. 


\section{Acknowledgement}

First of all, I would like to thank my supervisor, László Sághy, for the patient guidance, encouragement and advice he has provided throughout my time as his $\mathrm{PhD}$ fellow.

I'm especially grateful to my supervisors in Philadelphia, Dr. Yuchi Han, Dr. David J. Callans and Dr. Francis E. Marchlinski who have provided me with the opportunity to perform my work in their departments and for their support and cooperation in my scientific work.

I greatly acknowledge the continous encouragement of Professor Tamás Forster who provided me with the opportunity to begin my work at the $2^{\text {nd }}$ Department of Internal Medicine and Cardiology Center.

I would like to thank Dr. Larraitz Gaztanaga, who as a good friend was always willing to help and give her best suggestions.

I'm thankful to all collegues, all nurses and secretaries in the EP Lab for their support.

Last, but not least, I'm most indebted to my family for their understanding, support, patience and love. 